Article

\title{
Geometry of Fisher Information Metric and the Barycenter Map ${ }^{\dagger}$
}

\author{
Mitsuhiro Itoh ${ }^{1}$ and Hiroyasu Satoh ${ }^{2}$ \\ ${ }^{1}$ Institute of Mathematics, University of Tsukuba, 1-1-1, Ten-noudai, Tsukuba, 305-8571, Japan \\ ${ }^{2}$ Nippon Institute of Technology, Saitama, 345-8501, Japan; E-Mail: hiroyasu@ @it.ac.jp \\ $\dagger$ This paper is an extended version of our paper published in proceedings, 34th International Workshop \\ on Bayesian Inference and Maximum Entropy Methods in Science and Engineering, 21-26 \\ September 2014, Château Clos Lucé, Amboise, France. \\ * Author to whom correspondence should be addressed; E-Mail: itohm@ @ath.tsukuba.ac.jp; \\ Tel.: +81-29-853-4369.
}

Received: 28 January 2015 / Accepted: 13 March 2015 / Published: 30 March 2015

\begin{abstract}
Geometry of Fisher metric and geodesics on a space of probability measures defined on a compact manifold is discussed and is applied to geometry of a barycenter map associated with Busemann function on an Hadamard manifold $X$. We obtain an explicit formula of geodesic and then several theorems on geodesics, one of which asserts that any two probability measures can be joined by a unique geodesic. Using Fisher metric and thus obtained properties of geodesics, a fibre space structure of barycenter map and geodesical properties of each fibre are discussed. Moreover, an isometry problem on an Hadamard manifold $X$ and its ideal boundary $\partial X$ - for a given homeomorphism $\Phi$ of $\partial X$ find an isometry of $X$ whose $\partial X$-extension coincides with $\Phi$-is investigated in terms of the barycenter map.
\end{abstract}

Keywords: Fisher metric; probability measure; geodesic; Busemann function; barycenter

PACS classifications: $02.40 . K y ; 02.50 .-\mathrm{r}$ 


\section{Introduction}

The aim of this article is to deal with two subjects related with information geometry. One is Fisher metric $G$ defined on a space $\mathcal{P}(M)$ of probability measures having continuous positive density function over a connected, compact manifold $M$, and another one is barycenter map from $\mathcal{P}(\partial X)$ to an Hadamard manifold $X$, where $\partial X$ is the ideal boundary of $X$. This article is an extended version of [1] presented at MaxEnt 2014, Amboise, France.

The Fisher metric $G$, remarkably important in information geometry, is defined in a natural way. The metric $G$ is push-forward invariant, and has an explicit formula of Levi-Civita connection and its sectional curvature is constant $1 / 4$, as shown in [2] by T. Friedrich.

Before introducing main results, we will explain motivation and background of our study.

An $n$-dimensional Hadamard manifold $(X, g)$ is diffeomorphic to $\mathbf{R}^{n}$, and hence to an open ball $D^{n}$, whose actual boundary is $S^{n-1}$. $X$ admits also the ideal boundary $\partial X$ as a quotient space of oriented geodesics on $X$. Then, we are able to consider Dirichlet problem at boundary $\partial X$; given a $f \in C^{0}(\partial X)$, find a solution $u=u(x)$ on $X$ satisfying $\Delta u=0,\left.u\right|_{\partial X}=f$. Using the fundamental solution $P=P(x, \theta)$, called Poisson kernel, when its existence is guaranteed, the solution is described as

$$
u(x)=\int_{\theta \in \partial X} P(x, \theta) d \theta, \quad x \in X .
$$

Refer to [3] for precise definition of Poisson kernel. We obtain then a probability measure $P(x, \theta) d \theta$ on $\partial X$ parametrized in $x \in X$ and have a map, called Poisson kernel map $\Theta: X \rightarrow \mathcal{P}(\partial X)$.

Theorem 1 ([4-6]). Let $(X, g)$ be an n-dimensional Damek-Ricci space. Then the map $\Theta$ is homothetic with respect to the Fisher metric $G$ and $g ; \Theta^{*} G=\frac{Q}{n} g$ where $Q$ is volume entropy of $(X, g)$. Further $\Theta$ is a harmonic map.

Here, for volume entropy $Q$ refer to $\S 4$. The quantity $Q$ is an invariant of Riemannian geometry which is closely related to the topological entropy of geodesic flow $([7,8])$. Refer to [9] with respect to volume entropy treated in a framework of information geometry.

In the theorem a Damek-Ricci space is a solvable Lie group of a left invariant metric, one dimensional extension of a generalized Heisenberg group. Refer to [10] for details. A Damek-Ricci space is a harmonic, Einstein Hadamard manifold and any rank one symmetric space of non-compact type, namely hyperbolic spaces over the real numbers $\mathbf{R}$, the complex numbers $\mathbf{C}$, the quaternions $\mathbf{H}$ and 16-dimensional one over Cayley numbers $\mathbf{O}$ are also Damek-Ricci spaces. With respect to Theorem 1, we have the following theorem.

Theorem 2 ([5]). Let $(X, g)$ be an Hadamard manifold which is equipped with Poisson kernel $P(x, \theta)$. Assume that the map $\Theta: X \rightarrow \mathcal{P}(\partial X)$ is homothetic; $\Theta^{*} G=C g, C>0$, and harmonic with respect to the metrics $G$ and $g$. Then $(X, g)$ is asymptotically harmonic and satisfies visibility axiom. Moreover, $C=Q / n$ and the Poisson kernel has the form $P(x, \theta)=\exp \left\{-Q B_{\theta}(x)\right\}$ in terms of Busemann function $B_{\theta}$ on $X$.

The terminology with respect to asymptotical harmonicity, visibility axiom and Busemann function will be explained in the subsequent sections. 
Remark that the equality $C=Q / n$ is derived from asymptotical formula related with mean curvature of geodesic spheres and mean curvature of corresponding horospheres, level hypersurfaces of Busemann function ([11]).

With respect to these theorems we are interested in characterization of Damek-Ricci space from information geometry, especially from a viewpoint of Fisher metric $G$, since a Damek-Ricci space is a counterexample of Lichnerowicz conjecture of non-compact version ([12]) and its characterization is only given by Heber in [13] by Lie group theory argument. By approaching from a viewpoint of the ideal boundary $\partial X$, we focus on barycenter of probability measures on $\partial X$ with respect to Busemann function and shed a light on information geometry of barycenter map bar : $\mathcal{P}(\partial X) \rightarrow X$.

\section{Main Results and Conclusive Remarks}

Before entering into the detailed argument, we give an outline of main results and remarks.

In Section 3 we deal with several results on Fisher metric $G$ and also on geodesic, a basic notion of geometry, defined on $(\mathcal{P}(M), G)$. We give a formula of geodesic $\mu(t)=\exp _{\mu} t \tau$ on $\mathcal{P}(M)$ in a simple form (Theorem 9);

$$
\mu(t)=\left(\cos \frac{t}{2}+\sin \frac{t}{2} \frac{d \tau}{d \mu}(x)\right)^{2} \mu, \quad x \in M
$$

for an initial condition; $\mu(0)=\mu, \dot{\mu}(0)=\tau\left(|\tau|_{G, \mu}=1\right)$. Here, $(d \tau / d \mu)(x)$ denotes Radon-Nikodym derivative of $\tau$ with respect to $\mu$. From this, it is concluded in Corollary 2 that any geodesic is periodic, of period $2 \pi$, while not definable over R. Moreover, from this formula which is an improvement of the formula given by T. Friedrich ([2]) we obtain

Theorem 3. Let $\mu$ and $\mu^{*}$ be arbitrary distinct probability measures in $\mathcal{P}(M)$. Then, a curve $t \in \mathbf{R} \mapsto$ $\mu(t) \in \mathcal{P}(M)$ defined by

$$
\mu(t)=\exp _{\mu} t \tau=\left(\cos \frac{t}{2}+\sin \frac{t}{2} \frac{d \tau}{d \mu}(x)\right)^{2} \mu
$$

is a unique geodesic such that $\mu(0)=\mu$ and $\mu(\ell)=\mu^{*}$. Here $\ell=\ell\left(\mu, \mu^{*}\right)$ is defined by (4) and $\tau$ is a unit tangent vector at $\mu$ given by

$$
\tau=\frac{1}{\sin \frac{\ell}{2}}\left(\sqrt{\frac{d \mu^{*}}{d \mu}}(x)-\int_{y \in M} \sqrt{\frac{d \mu^{*}}{d \mu}}(y) d \mu(y)\right) \mu(x) .
$$

This theorem asserts that any $\mu, \mu^{*}, \mu \neq \mu^{*}$ can be joined by a unique geodesic. The quantity $\ell=\ell\left(\mu, \mu^{*}\right), 0<\ell<\pi$ is defined as

$$
\cos \frac{\ell}{2}=\int_{x \in M} \sqrt{\frac{d \mu^{*}}{d \mu}}(x) d \mu(x)
$$

giving an apparent length of a geodesic joining $\mu$ and $\mu^{*}$. Notice that the RHS is an $f$-divergence-like quantity with respect to $f(u)=\sqrt{u}$ (refer to [14]). 
Another main subject is information geometry of barycenter map by applying results of Fisher information geometry, thus obtained in Section 3. Related results on barycenter map will be explained in Section 4 and Section 5.

Let $(X, g)$ be an Hadamard manifold with a Riemannian metric $g$, a simply connected, complete Riemannian manifold of non-positive curvature. Then, it admits the ideal boundary $\partial X$ and by using a probability measure defined on $\partial X$, we consider a function, a $\mu$-average Busemann function $\mathbf{B}_{\mu}: X \rightarrow \mathbf{R}$

$$
\mathbf{B}_{\mu}(x)=\int_{\theta \in \partial X} B_{\theta}(x) d \mu(\theta)
$$

whose critical point is called a barycenter of a probability measure $\mu$ so that we have a map, barycenter map, from a space $\mathcal{P}(\partial X)$ of probability measures on $\partial X$ to an Hadamard manifold $X$. Here, the integrand is a normalized Busemann function (for its detailed argument see Section 4).

Recall, here, an original definition of a barycenter, a center of mass, as follows. Let $y_{1}, \ldots, y_{n}$ be points of a Euclidean space $\mathbf{R}^{3}$ and $\mu_{1}, \ldots, \mu_{n}$ be non-negative real numbers satisfying $\sum_{i} \mu_{i}=1$. A point $p$ of $\mathbf{R}^{3}$ is called a barycenter of $y_{i}, i=1, \ldots, n$ of weights $\mu_{i}, i=1, \ldots, n$, when $p$ satisfies

$$
p=\sum_{i=1}^{n} \mu_{i} y_{i} \quad \text { or } \quad \sum_{i=1}^{n} \mu_{i}\left(y_{i}-p\right)=0 .
$$

A barycenter is defined also by a critical point of a function on $\mathbf{R}^{3} ; f: \mathbf{R}^{3} \rightarrow \mathbf{R} ; f(q)=$ $\sum_{i=1}^{n} \mu_{i} d^{2}\left(q, y_{i}\right)$.

This definition of barycenter for a finite points of $\mathbf{R}^{3}$ with weights with respect to the square-distance can be generalized as one for points of $\mathbf{R}^{3}$ distributed continuously over a bounded set $D$ of $\mathbf{R}^{3}$;

$$
f: \mathbf{R}^{3} \rightarrow \mathbf{R} ; \quad f(q)=\int_{\mathbf{R}^{3}} d^{2}(q, x) \mu(x) d x,
$$

where $\mu=\mu(x)$ is a non-negative function with $\operatorname{supp}(\mu) \subset D$ satisfying $\int_{\mathbf{R}^{3}} \mu(x) d x=1$. A critical point of $f$ can be regarded as a barycenter of a probability measure $\mu(x) d x$. A famous theorem of E. Cartan is regarded as a barycenter theorem ([15]). A choice of testing function $d^{2}(x, y)$ is not essential. Convexity of testing function is crucial in a theory of barycenter. In our study we deal with barycenter with respect to Busemann function, a convex testing function, by following the idea of Douady, Earle ([16]) and Besson, Courtois and Gallot ([8,17]). Refer to [18,19] for studies and results on barycenter of square-distance and of distance over a Riemannian manifold. Refer also to [20] in this direction, which is a reference comment due to Professor M. Gromov at the conference.

In our situation, the existence of barycenter for any $\mu \in \mathcal{P}(\partial X)$ is assured in Theorem 12, when $(X, g)$ satisfies visibility axiom (for precise definition see Definition 4 and refer to [21]) and Busemann function $B_{\theta}(x)$ on $X$ is continuous with respect to $\theta \in \partial X$. Uniqueness of barycenter for any $\mu$ is assured, when, for some $\mu_{0}$, average Hessian $\nabla d \mathbf{B}_{\mu_{0}}$ of $\mathbf{B}_{\mu_{0}}$ is positive definite everywhere on $X$ (Proposition 6). Thus, we have the barycenter map bar : $\mathcal{P}(\partial X) \rightarrow X ; \mu \mapsto y$, by assigning to $\mu$ a barycenter point $y$ of $\mu$. This map turns out to be surjective, when $(X, g)$ admits Busemann-Poisson kernel $P(x, \theta)=\exp \left\{-Q B_{\theta}(x)\right\}$, Poisson kernel of Busemann type. Denote by $\mu_{x}$ the probability 
measure $P(x, \theta) d \theta$. Then, $\operatorname{bar}\left(\mu_{x}\right)=x$ for any $x \in X$. Busemann-Poisson kernel ensures also the uniqueness of barycenter for any $\mu$ from the identity (see Theorem 14)

$$
\left(\nabla d \mathbf{B}_{\mu_{x}}\right)_{x}(u, v)=Q G_{\mu_{x}}\left(\nu_{x}^{\mu_{x}} u, \nu_{x}^{\mu_{x}} v\right), u, v \in T_{x} X, x \in X
$$

where, $G_{\mu_{x}}$ is Fisher metric at the tangent space $T_{\mu_{x}} \mathcal{P}(\partial X)$, and $\nu_{x}^{\mu_{x}}: T_{x} X \rightarrow T_{\mu_{x}} \mathcal{P}(\partial X)$ is an injective linear map associated to $\mu$ and a point $x=\operatorname{bar}(\mu)$ (for its definition see Section 4).

The map bar $: \mathcal{P}(\partial X) \rightarrow X$, being surjective gives us a projection of a fibre space whose total space is $\mathcal{P}(\partial X)$ and base space is $X$ with fibres $\left\{\operatorname{bar}^{-1}(x) ; x \in X\right\}$. The image of the linear map $\nu_{x}^{\mu}$ for $\mu$ and $x=\operatorname{bar}(\mu)$ yields a subspace of $T_{\mu} \mathcal{P}(\partial X)$, normal to $T_{\mu} \mathrm{bar}^{-1}(x)$, the subspace tangent to a fibre $\operatorname{bar}^{-1}(x)$ so that $T_{\mu} \mathcal{P}(\partial X)$ splits into a $G$-orthogonal direct sum (Theorem 15);

$$
T_{\mu} \mathcal{P}(\partial X)=T_{\mu} \operatorname{bar}^{-1}(x) \oplus \operatorname{Im} \nu_{x}^{\mu}
$$

$T_{\mu} \operatorname{bar}^{-1}(x)$ and $\operatorname{Im} \nu_{x}^{\mu}$ are the vertical, horizontal subspaces of $T_{\mu} \mathcal{P}(\partial X)$, respectively. Here, $\operatorname{dim} \operatorname{Im} \nu_{x}^{\mu}=\operatorname{dim} X$. Remark that the fibration asserted here is infinitesimal.

Each fibre $\operatorname{bar}^{-1}(x), x \in X$ is a path-connected submanifold of $\mathcal{P}(\partial X)$. Its geometry is investigated in terms of the second fundamental form;

$$
H_{\mu}: T_{\mu} \operatorname{bar}^{-1}(x) \times T_{\mu} \operatorname{bar}^{-1}(x) \rightarrow \operatorname{Im} \nu_{x}^{\mu} ; \quad H_{\mu}\left(\tau, \tau_{1}\right)=\left(\nabla_{\tau} \tau_{1}\right)^{\perp}
$$

which is the normal component of $\nabla_{\tau} \tau_{1}$, the covariant derivative of $\tau_{1}$ in direction to $\tau$ with respect to the Levi-Civita connection $\nabla$. Refer to [22,23] for definition of the second fundamental form. Applying the results concerning geodesics on $\mathcal{P}(\partial X)$ given in Section 3 to a submanifold $\operatorname{bar}^{-1}(x)$, we are able to determine a geodesic $\mu(t)=\exp _{\mu} t \tau$ which is entirely contained in $\operatorname{bar}^{-1}(x)$ as

Theorem 4. Let $\mu(t)=\exp _{\mu} t \tau$ be a unit speed geodesic, of $\mu(0)=\mu, \dot{\mu}(0)=\tau,|\tau|_{G, \mu}=1$. Then, $\mu(t)$ lies entirely on fibre $\operatorname{bar}^{-1}(x)$ if and only if, $\mu \in \operatorname{bar}^{-1}(x), \tau \in T_{\mu} \operatorname{bar}^{-1}(x)$ and $H_{\mu}(\tau, \tau)=0$.

Remark that the equation $H_{\mu}(\tau, \tau)=0$ on $\tau$ is written down in a manner of information geometry as $\int_{\theta \in \partial X}\left(d B_{\theta}\right)_{x}(u)(d \tau / d \mu)^{2}(\theta) d \mu(\theta)=0$.

Moreover, by applying Theorem 11 in Section 3, it is possible to assert the following theorem.

Theorem 5. Let $\mu, \mu^{*} \in \operatorname{bar}^{-1}(x)$. Then, a geodesic joining $\mu$ and $\mu^{*}$ is contained completely in the same fibre $\operatorname{bar}^{-1}(x)$ if and only if $\mu$ and $\mu^{*}$ fulfill

$$
\int_{\theta \in \partial X}\left(d B_{\theta}\right)_{x}(u) \sqrt{\frac{d \mu^{*}}{d \mu}}(\theta) d \mu=0
$$

for any $u \in T_{x} X$.

Let $\phi$ be an isometry of an Hadamard manifold $(X, g)$. Then, a $\mu$-average Busemann function $\mathbf{B}_{\mu}$ satisfies a cocycle formula with respect to $\phi$;

$$
\mathbf{B}_{\mu}\left(\phi^{-1} x\right)=\mathbf{B}_{\left(\hat{\phi}_{\sharp)} \mu\right.}(x)+\mathbf{B}_{\mu}\left(\phi^{-1} x_{o}\right), \quad x \in X, \mu \in \mathcal{P}(\partial X),
$$


where $\phi^{-1}$ is the inverse of $\phi$, and $\hat{\phi}: \partial X \rightarrow \partial X$ is a $\partial X$-extension of $\phi$ and $\hat{\phi}_{\sharp}$ is a push-forward induced by $\hat{\phi}$. See Theorem 18 . From this formula we have

$$
\operatorname{bar}\left(\hat{\phi}_{\sharp} \mu\right)=\phi(\operatorname{bar}(\mu)), \quad \mu \in \mathcal{P}(\partial X)
$$

from which each fibre $\operatorname{bar}^{-1}(x)$ is mapped by $\hat{\phi}_{\sharp}$ to a fibre $\operatorname{bar}^{-1}(\phi x)$ over $\phi x$.

By the aid of information geometry we are able to apply above results to an isometry problem; given a homeomorphism $\Phi$ of $\partial X$, find an isometry $\phi$ of $(X, g)$ whose $\partial X$-extension coincides with $\Phi$. With respect to this problem we consider a bijective map $\phi$ of $X$ satisfying $\operatorname{bar}\left(\Phi_{\sharp} \mu\right)=\phi(\operatorname{bar}(\mu))$ for any $\mu \in \mathcal{P}(\partial X)$ ( we call such a map $\phi$ as barycentrically associated to $\Phi$ ).

The following theorem gives us an answer to this problem, even partial, provided there exists a cross section of the fibre space bar $: \mathcal{P}(\partial X) \rightarrow X$ enjoying commutativity properties.

Theorem 6. Let $\varphi: X \rightarrow X$ be a $C^{1}$-map barycentrically associated to a homeomorphism $\Phi: \partial X \rightarrow$ $\partial X$. Assume that there exists a cross section $\Sigma: X \rightarrow \mathcal{P}(\partial X)$ of the fibre space bar $: \mathcal{P}(\partial X) \rightarrow X, a$ map satisfying bar $\circ \Sigma=i d_{X}$ such that a fibrewise diagram commutes

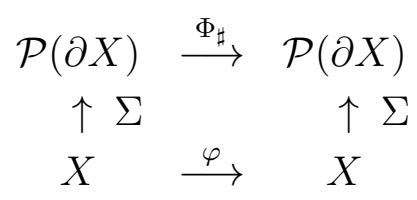

and a diagram of tangent space level commutes

$$
\begin{array}{ccc}
T_{x} X & \stackrel{\nu_{x}^{\mu_{x}}}{\longrightarrow} & T_{\mu_{x}} \mathcal{P}(\partial X) \\
\downarrow\left(\varphi_{*}\right)_{x} & & \downarrow \Phi_{\sharp} \\
T_{\varphi x} X & \stackrel{\nu_{\varphi x}^{\mu_{\varphi x}}}{\longrightarrow} & T_{\mu_{\varphi x}} \mathcal{P}(\partial X)
\end{array}
$$

where we denote by $\mu_{x}:=\Sigma(x) \in \mathcal{P}(\partial X)$. Then, $\varphi$ is an isometry of $(X, g)$ and $\partial X$-extension $\hat{\varphi}$ of $\varphi$ coincides with $\Phi$.

A particularly significant cross section $\Sigma$ is given by a Poisson kernel map; $\Theta: X \rightarrow \mathcal{P}(\partial X)$; $x \mapsto P(x, \theta) d \theta=\exp \left\{-Q B_{\theta}(x)\right\} d \theta$, where $P(x, \theta)$ is a Busemann-Poisson kernel on $(X, g)$. The differential map $\left(\Theta_{*}\right)_{x}$ of $\Theta$ fulfills $\left(\Theta_{*}\right)_{x}(u)=-Q \nu_{x}^{\mu_{x}}(u), u \in T_{x} X$ in terms of the linear map $\nu_{x}^{\mu_{x}}$, $\mu_{x}:=P(x, \theta) d \theta$, so that we have

Corollary 1 ([24]). Let $\Phi: \partial X \rightarrow \partial X$ be a homeomorphism of $\partial X$ and $\varphi: X \rightarrow X$ be a $C^{1}$-map. Assume the following diagram commutes with respect to Poisson kernel map $\Theta$;

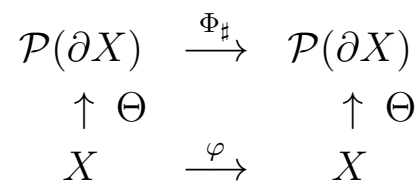

Then, $\varphi$ is an isometry of $(X, g)$ and its $\partial X$-extension coincides with $\Phi$ of $\partial X$.

Theorem 6 is a generalization of Corollary 1, a main result of [24,25]. 
The article is organized as follows. In Section 3 we introduce basic notions of information geometry of a space $\mathcal{P}(M)$ of probability measures on a compact manifold $M$ and define Fisher metric $G$ on it. We show several useful theorems on the Levi-Civita connection and geodesics on $\mathcal{P}(M)$ with detailed proofs. We derive in Section 4 fundamental properties of Busemann function on an Hadamard manifold, preliminarily. By using them, we investigate existence and uniqueness of barycenter of a probability measure, following a proof given in [8]. We define the barycenter map and develop information geometry of this map. A fibration theorem is similar to our earlier paper [25]. However, geodesical arguments on fibres develop further the arguments of [25], by applying the results of geodesics on $\mathcal{P}(M)$ in Section 3. Finally, in Section 5, we treat an isometry problem for an Hadamard manifold and give a proof of Theorem 6.

\section{A Space of Probability Measures and Fisher Metric}

\subsection{A Space of Probability Measures}

Let $M$ be a connected, compact smooth manifold. Let $\mathcal{B}(M)$ be the family of Borel sets of $M$. Here $\mathcal{B}(M)$ is a family of subsets of $M$ which satisfies the following; (i) $\mathcal{B}(M)$ is a $\sigma$-family of $M$, (ii) every open subset of $M$ is an element of $\mathcal{B}(M)$ and (iii) if $\mathcal{F}$ is a family of subsets of $M$ satisfying (i), (ii), then $\mathcal{F} \subset \mathcal{B}(M)$.

A function $P: \mathcal{B}(M) \rightarrow \mathbf{R}$ is called a probability measure of a measurable space $(M, \mathcal{B}(M))$, or a probability measure on $M$, when $P$ satisfies

(i) $P(A) \geq 0$ for any $A \in \mathcal{B}(M), P(M)=1$ and $P(\emptyset)=0$.

(ii) Let $\left\{E_{j} \mid j=1, \ldots,\right\}$ be a countable sequence of sets of $\mathcal{B}(M)$ satisfying $E_{i} \cap E_{j}=\emptyset$ for any $i, j, i \neq j$. Then

$$
P\left(\bigcup_{i=1}^{\infty} E_{i}\right)=\sum_{i=1}^{\infty} P\left(E_{i}\right) .
$$

A smooth manifold $M$, even in an unorientable case, admits a measure induced by the volume measure of $M$. We normalize this measure and denote this normalized measure by $d \theta$. The measure $d \theta$ is a probability measure on $M$.

For example, let $M=S^{n-1}=\left\{x \in \mathbf{R}^{n} ;|x|=1\right\}$ be a unit $(n-1)$-sphere and let $d \omega$ be the standard $(n-1)$-spherical volume measure on $S^{n-1}$. Then $d \theta=\left(1 / A_{n-1}\right) d \omega$ is the normalized measure, where $A_{n-1}$ is the volume of $S^{n-1}$.

Let $\mu, \mu_{1}$ be probability measures on $M . \mu$ is called to be absolutely continuous with respect to $\mu_{1}$, if $\mu(A)=0$ for any $A \in \mathcal{B}(M)$, whenever $\mu_{1}(A)=0$.

Let $\mu$ be a probability measure on $M$, absolutely continuous with respect to $d \theta$. Then, from Radon-Nikodym theorem ([26]) there exists a $p \in L^{1}(M, d \theta)$ such that $\mu$ is represented as $\mu=p d \theta$, namely $\mu$ satisfies

$$
\mu(A)=\int_{x \in A} p(x) d \theta(x), \quad \forall A \in \mathcal{B}(M) .
$$

The probability density function $p=p(x)$ is called Radon-Nikodym derivative of $\mu$ with respect to $d \theta$, written by $p=d \mu / d \theta$. 
We denote by $\mathcal{P}(M)$ a space of probability measures $\mu$ on $M, d \theta$-absolutely continuous (denoted by $\mu \ll d \theta)$ such that $\mu$ has a positive continuous density function $p=p(x)$, i.e., $p \in C^{0}(M), p(x)>0$ for any $x \in M$.

A manifold $M$ admits an $L^{2}$-function space $L^{2}(M, d \theta)$ as

$$
L^{2}(M, d \theta)=\left\{h: M \rightarrow \mathbf{R} ; \int_{M} h^{2}(x) d \theta(x)<\infty\right\} .
$$

We notice that there exists a natural embedding

$$
\rho: \mathcal{P}(M) \rightarrow L^{2}(M, d \theta) ; \quad \mu=p d \theta \mapsto \sqrt{p}=\sqrt{\frac{d \mu}{d \theta}}
$$

By using this embedding we induce a topology on $\mathcal{P}(M)$. Remark that a sequence $\left\{\mu_{i}\right\}$ of $\mathcal{P}(M)$ does not necessarily admit a limit inside $\mathcal{P}(M)$.

Let $\mu, \mu_{1}$ be probability measures in $\mathcal{P}(M)$. Then we can join $\mu$ and $\mu_{1}$ by a path $\mu(t)=(1-t) \mu+t \mu_{1}$, $t \in[0,1]$ inside $\mathcal{P}(M)$.

Differentiate $\mu(t)$ as a curve in $\mathcal{P}(M)$ to have

$$
\left.\frac{d}{d t}\left((1-t) \mu+t \mu_{1}\right)\right)=\mu_{1}-\mu
$$

which is a measure on $M$, represented as $\mu_{1}-\mu=\left(p_{1}-p\right) d \theta$ satisfying

$$
\int_{M} d\left(\mu_{1}-\mu\right)=0
$$

Based on this fact, a tangent space $T_{\mu} \mathcal{P}(M)$ of $\mathcal{P}(M)$ at $\mu$ is defined as

$$
T_{\mu} \mathcal{P}(M)=\left\{\tau=q(x) d \theta(x) ; q \in C^{0}(M), \int_{M} q(x) d \theta(x)=0\right\}
$$

Notice that the RHS (right hand side) of (9) is independent of $\mu$. So, if we denote by $\mathcal{V}$ the RHS of (9), then $\mathcal{V}$ is an infinite dimensional vector space and an arbitrary $\tau \in \mathcal{V}$ induces at any $\mu \in \mathcal{P}(M)$ a curve $\mu+t \tau \in \mathcal{P}(M)$ for $t \in(-\varepsilon, \varepsilon)$ with a sufficiently small $\varepsilon$.

We define a curve $c:(a, b) \rightarrow \mathcal{P}(M)$ as

$$
c(t)=p(x, t) d \theta
$$

where $p(x, t)$ is of $C^{1}$ in $t$ for any fixed $x \in M$. So, $c=c(t)$ has velocity vector field along $c$

$$
\frac{d c}{d t}(t)=\frac{\partial}{\partial t} p(x, t) d \theta \in T_{c(t)} \mathcal{P}(M), \quad t \in(a, b)
$$

\subsection{Fisher Metric}

Definition 1. A positive definite inner product $G_{\mu}$ on $T_{\mu} \mathcal{P}(M)$ at $\mu \in \mathcal{P}(M)$ is defined as

$$
G_{\mu}: T_{\mu} \mathcal{P}(M) \times T_{\mu} \mathcal{P}(M) \rightarrow \mathbf{R} ; \quad G_{\mu}\left(\tau, \tau_{1}\right)=\int_{x \in M} \frac{d \tau}{d \mu}(x) \frac{d \tau_{1}}{d \mu}(x) d \mu(x) .
$$

A family $G=\left\{G_{\mu} \mid \mu \in \mathcal{P}(M)\right\}$ is called Fisher metric. $\sqrt{G_{\mu}(\tau, \tau)}$ is denoted by $|\tau|_{G, \mu}$. 
The Fisher metric is a generalization of Fisher information matrices appeared in parametric models. The following is one of remarkable properties of the Fisher metric $G$.

Let $\Phi: M \rightarrow M$ be a homeomorphism of $M$. Then, $\Phi$ induces a push-forward

$$
\Phi_{\sharp}: \mathcal{P}(M) \rightarrow \mathcal{P}(M) ; \quad\left(\Phi_{\sharp}(\mu)\right)(A):=\mu\left(\Phi^{-1}(A)\right), \quad A \in \mathcal{B}(M) .
$$

Here $\mu(A)=\int_{x \in A} d \mu(x)$. See [27]. We represent (10) in an integral form as

$$
\int_{x \in A} q(x) d\left(\Phi_{\sharp} \mu\right)(x)=\int_{x \in \Phi^{-1}(A)} q(\Phi(x)) d \mu(x)
$$

for any measurable function $q: M \rightarrow \mathbf{R}$.

When $\Phi$ is self-diffeomorphism of $M, \Phi_{\sharp} \mu$ coincides with $\left(\Phi^{-1}\right)^{*} \mu$, the pull-back of $\mu$ by the inverse diffeomorphism $\Phi^{-1}$. Notice that

$$
(\Phi \circ \Psi)_{\sharp}=\Phi_{\sharp} \circ \Psi_{\sharp}
$$

for homeomorphisms $\Phi, \Psi$ of $M$ and that the push-forward $\Phi_{\sharp}: \mathcal{P}(M) \rightarrow \mathcal{P}(M)$ has differential map

$$
\left(d \Phi_{\sharp}\right)_{\mu}: T_{\mu} \mathcal{P}(M) \rightarrow T_{\Phi_{\sharp}} \mathcal{P}(M) ; \quad\left(d \Phi_{\sharp}\right)_{\mu}(\tau)=\Phi_{\sharp}(\tau) .
$$

Here $\Phi_{\sharp} \tau$ is defined similarly as (10). In fact, we have

$$
\left(d \Phi_{\sharp}\right)_{\mu}(\tau)=\left.\frac{d}{d t}\right|_{t=0}\left(\Phi_{\sharp}(\mu+t \tau)\right)=\left.\frac{d}{d t}\right|_{t=0}\left(\Phi_{\sharp}(\mu)+t \Phi_{\sharp}(\tau)\right)=\Phi_{\sharp}(\tau) .
$$

Theorem 7 ([2]). Let $\Phi_{\sharp}$ be a push-forward. Then it acts on $\mathcal{P}(M)$ isometrically with respect to the Fisher metric G. Namely,

$$
G_{\Phi_{\sharp}}\left(\Phi_{\sharp} \tau, \Phi_{\sharp} \tau_{1}\right)=G_{\mu}\left(\tau, \tau_{1}\right), \quad \forall \tau, \tau_{1} \in T_{\mu} \mathcal{P}(M), \forall \mu \in \mathcal{P}(M) .
$$

Proof. We write $\mu=p(x) d \theta(x)$ and $\tau=q(x) d \theta(x), \tau_{1}=q_{1}(x) d \theta(x)$. Set $\sigma=\Phi_{\sharp} \mu$. We have then from definition of push-forward

$$
\sigma=p\left(\Phi^{-1}(x)\right) \Phi_{\sharp} d \theta(x) .
$$

This follows in fact from

$$
\begin{aligned}
\int_{M} h(x) d\left(\Phi_{\sharp} \mu\right)(x) & =\int_{M} h(\Phi(x)) d \mu(x)=\int_{M} h(\Phi(x)) p(x) d \theta(x) \\
& =\int_{M}\left(h \times\left(p \circ \Phi^{-1}\right)\right)(\Phi(x)) d \theta(x)
\end{aligned}
$$

for any measurable function $h$ on $M$, which, by definition of push-forward, coincides with $\int_{M} h(x)(p \circ$ $\left.\Phi^{-1}\right)(x) \Phi_{\sharp} d \theta(x)$ and the above is obtained.

In a similar way to (11) we have

$$
\Phi_{\sharp} \tau=q\left(\Phi^{-1}(x)\right) \Phi_{\sharp} d \theta(x), \quad \Phi_{\sharp} \tau_{1}=q_{1}\left(\Phi^{-1}(x)\right) \Phi_{\sharp} d \theta(x),
$$


so that

$$
\begin{aligned}
G_{\sigma}\left(\Phi_{\sharp} \tau, \Phi_{\sharp} \tau_{1}\right) & =\int_{x \in M}\left(\frac{d \Phi_{\sharp} \tau}{d \sigma}\right)(x)\left(\frac{d \Phi_{\sharp} \tau_{1}}{d \sigma}\right)(x) d \sigma(x) \\
& =\int_{x \in M} \frac{q\left(\Phi^{-1}(x)\right)}{p\left(\Phi^{-1}(x)\right)} \frac{q_{1}\left(\Phi^{-1}(x)\right)}{p\left(\Phi^{-1}(x)\right)} d\left(\Phi_{\sharp} \mu\right)(x) \\
& =\int_{x \in M} \frac{d \tau}{d \mu}\left(\Phi^{-1}(x)\right) \frac{d \tau_{1}}{d \mu}\left(\Phi^{-1}(x)\right) d\left(\Phi_{\sharp} \mu\right)(x) .
\end{aligned}
$$

Set $F(x)=\frac{d \tau}{d \mu}\left(\Phi^{-1}(x)\right) \frac{d \tau_{1}}{d \mu}\left(\Phi^{-1}(x)\right)$ and write the above as

$$
\int_{M} F(x) d\left(\Phi_{\sharp} \mu\right)(x)=\int_{M} F(\Phi(x)) d \mu(x)=\int_{M} \frac{d \tau}{d \mu}(x) \frac{d \tau_{1}}{d \mu}(x) d \mu(x)
$$

which is the inner product $G_{\mu}\left(\tau, \tau_{1}\right)$ of $\tau$ and $\tau_{1}$ at $\mu$.

Note. The following is known. For any $\mu \in \mathcal{P}(M)$ there exists a homeomorphism $\Phi: M \rightarrow M$ satisfying $\mu=\Phi_{\sharp} d \theta$ (refer to [28,29]). This fact implies that the action of $\operatorname{Homeo}(M)$, which is the group of homeomorphisms on $M$, on the space $\mathcal{P}(M)$ is isometric and transitive.

Remark 1. The embedding $\rho$ given in (8) satisfies $\rho^{*}\langle\cdot, \cdot\rangle_{L^{2}}=\frac{1}{4} G$, that is,

$$
\left\langle\left(\rho_{*}\right)_{\mu} \tau,\left(\rho_{*}\right)_{\mu} \tau_{1}\right\rangle_{L^{2}}=\frac{1}{4} G_{\mu}\left(\tau, \tau_{1}\right), \quad \forall \tau, \tau_{1} \in T_{\mu} \mathcal{P}(M), \mu \in \mathcal{P}(M) .
$$

where $\langle\cdot, \cdot\rangle_{L^{2}}$ is the $L^{2}$-inner product of $L^{2}(M, d \theta)$;

$$
\left\langle f_{1}, f_{2}\right\rangle_{L^{2}}:=\int_{x \in M} f_{1}(x) f_{2}(x) d \theta(x), f_{1}, f_{2} \in L^{2}(M, d \theta) .
$$

\subsection{Levi-Civita Connection}

The Fisher metric provides the space $\mathcal{P}(M)$ a Riemannian metric as above and then induces on $\mathcal{P}(M)$ the Levi-Civita connection $\nabla$ and the Riemannian curvature tensor $R$. To derive their formulae we will introduce a constant vector fields on $\mathcal{P}(M)$.

Let $\tau \in \mathcal{V}$. Then, $\tau$ is considered as a constant vector field on $\mathcal{P}(M)$ by defining a vector field $\left\{\tau_{\mu} \mid \mu \in \mathcal{P}(M)\right\}, \tau_{\mu}=\left.\frac{d}{d t}\right|_{t=0} \mu(t) \in T_{\mu} \mathcal{P}(M)$, that is, $\tau$ is a velocity vector of a curve $\mu(t)=\mu+t \tau$ at $t=0$. Notice that an integral curve of a constant vector field $\tau$ passing through $\mu \in \mathcal{P}(M)$ is given by the curve $\mu(t)$.

Theorem 8. Let $\tau, \tau_{1}$ be constant vector fields on $\mathcal{P}(M)$. Then, the Levi-Civita connection of the Fisher metric $G$ at $\mu \in \mathcal{P}(M)$ is represented as

$$
\begin{aligned}
\nabla_{\tau} \tau_{1} & =-\frac{1}{2}\left(\frac{d \tau}{d \mu}(x) \frac{d \tau_{1}}{d \mu}(x)-G_{\mu}\left(\tau, \tau_{1}\right)\right) \mu \\
& =-\frac{1}{2}\left(\frac{d \tau}{d \mu}(x) \frac{d \tau_{1}}{d \mu}(x)-\int_{y \in M} \frac{d \tau}{d \mu}(y) \frac{d \tau_{1}}{d \mu}(y) d \mu(y)\right) \mu
\end{aligned}
$$

For this formula see [2]. 
Proof. Recall that on a Riemannian manifold $N$ with a Riemannian metric $g$ the Levi-Civita connection $\nabla$ of $g$ is an affine connection on $N$, that is, $\nabla$ is a bilinear map; $(Y, Z) \mapsto \nabla_{Y} Z$ satisfying

$$
\begin{aligned}
\nabla_{f Y} Z & =f \nabla_{Y} Z, \\
\nabla_{Y}(f Z) & =Y f \cdot Z+f \nabla_{Y} Z
\end{aligned}
$$

for smooth vector fields $Y, Z$ on $N$ and a smooth function $f$ on $N$, which satisfies $\left(\nabla_{Y} g\right)(Z, W)=0$ together with a symmetry condition, that is, the torsion tensor $T(Y, Z):=\nabla_{Y} Z-\nabla_{Z} Y-[Y, Z]$ vanishes. Then, the Levi-Civita connection $\nabla$ exists uniquely and one has Koszul's formula for $\nabla$

$g\left(\nabla_{Y} Z, W\right)=\frac{1}{2}\{Y g(Z, W)+Z g(W, Y)-W g(Y, Z)+g([Y, Z], W)-g([Z, W], Y)-g([Y, W], Z)\}$.

Here $Y, Z, W$ are smooth vector fields on $N$ ([22]).

We give a reference comment on a metric connection with non-trivial torsion, appeared in information geometry. A non-trivial torsion $T$ implies geometrically a breaking of the symmetry in connection coefficients; $\Gamma_{i j}^{k}=\Gamma_{j i}^{k}$. In a framework of classical parametric model there are very few study of a metric connection with non-trivial torsion. However, as far as the authors know, the $e$-connection developed in a quantum model has non-trivial torsion. Refer to Chapter 7 of [14] and references cited there.

Now we return back to our situation, that is, to the space $(\mathcal{P}(M), G)$ in which we have for constant vector fields $\tau, \tau_{1}$ and $\tau_{2}$

$$
G\left(\nabla_{\tau_{2}} \tau, \tau_{1}\right)=\frac{1}{2}\left\{\tau_{2} G\left(\tau, \tau_{1}\right)+\tau G\left(\tau_{1}, \tau_{2}\right)-\tau_{1} G\left(\tau, \tau_{2}\right)\right\}
$$

since $\left[\tau, \tau_{1}\right]=\left[\tau, \tau_{2}\right]=\left[\tau_{1}, \tau_{2}\right]=0$.

Let $\mu(t)=\mu+t \tau_{2}$ be a curve in $\mathcal{P}(M)$ of $\mu(0)=\mu$ and $\dot{\mu}(t)=\tau_{2}$. We have then

$$
\begin{aligned}
\left(\tau_{2}\right)_{\mu} G\left(\tau, \tau_{1}\right) & =\left.\frac{d}{d t}\right|_{t=0} G_{\mu(t)}\left(\tau, \tau_{1}\right) \\
& =\left.\frac{d}{d t}\right|_{t=0} \int_{M} \frac{d \tau}{d \mu(t)}(x) \frac{d \tau_{1}}{d \mu(t)}(x) d \mu(t)(x) \\
& =\left.\int_{M} \frac{\partial}{\partial t}\right|_{t=0}\left(\frac{d \tau}{d \mu(t)}(x) \frac{d \tau_{1}}{d \mu(t)}(x) d \mu(t)(x)\right)
\end{aligned}
$$

in which the integrand is

$$
\begin{aligned}
& \left.\frac{\partial}{\partial t}\right|_{t=0}\left(\frac{d \tau}{d \mu(t)}(x) \frac{d \tau_{1}}{d \mu(t)}(x) d \mu(t)(x)\right) \\
= & \left.\frac{\partial}{\partial t}\left(\frac{d \tau}{d \mu(t)}(x)\right)\right|_{t=0} \frac{d \tau_{1}}{d \mu}(x) d \mu(x)+\left.\frac{d \tau}{d \mu}(x) \frac{\partial}{\partial t}\left(\frac{d \tau_{1}}{d \mu(t)}(x)\right)\right|_{t=0} d \mu(x) \\
& +\left.\frac{d \tau}{d \mu}(x) \frac{d \tau_{1}}{d \mu}(x) \frac{\partial}{\partial t}(d \mu(t)(x))\right|_{t=0} .
\end{aligned}
$$

The partial derivative term becomes

$$
\left.\frac{\partial}{\partial t}\left(\frac{d \tau}{d \mu(t)}(x)\right)\right|_{t=0}=-\frac{d \tau}{d \mu}(x) \frac{d \tau_{2}}{d \mu}(x),
$$


since by calculation

$$
\begin{aligned}
\left.\frac{\partial}{\partial t}\left(\frac{d \tau}{d \mu(t)}(x)\right)\right|_{t=0} & =\left.\frac{\partial}{\partial t}\left(\frac{q(x)}{p(x)+t q_{2}(x)}\right)\right|_{t=0}=-\left.\frac{q(x) q_{2}(x)}{\left(p(x)+t q_{2}(x)\right)^{2}}\right|_{t=0} \\
& =-\frac{q(x) q_{2}(x)}{p^{2}(x)}=-\frac{d \tau}{d \mu}(x) \frac{d \tau_{2}}{d \mu}(x) .
\end{aligned}
$$

Similarly

$$
\left.\frac{\partial}{\partial t}\left(\frac{d \tau_{1}}{d \mu(t)}(x)\right)\right|_{t=0}=-\frac{d \tau_{1}}{d \mu}(x) \frac{d \tau_{2}}{d \mu}(x)
$$

Since

$$
\left.\frac{\partial}{\partial t}(d \mu(t)(x))\right|_{t=0}=d \tau_{2}(x)
$$

one obtains

$$
\begin{aligned}
\left(\tau_{2}\right)_{\mu} G\left(\tau, \tau_{1}\right)= & \int_{M}\left(-\frac{d \tau}{d \mu}(x) \frac{d \tau_{2}}{d \mu}(x)\right) \frac{d \tau_{1}}{d \mu}(x) d \mu(x) \\
& +\int_{M} \frac{d \tau}{d \mu}(x)\left(-\frac{d \tau_{1}}{d \mu}(x) \frac{d \tau_{2}}{d \mu}(x)\right) d \mu(x)+\int_{M}\left(\frac{d \tau}{d \mu}(x) \frac{d \tau_{1}}{d \mu}(x)\right) d \tau_{2}(x) .
\end{aligned}
$$

Here $d \tau_{2}(x)=\frac{d \tau_{2}}{d \mu}(x) d \mu(x)$. So,

$$
\left(\tau_{2}\right)_{\mu} G\left(\tau, \tau_{1}\right)=-\int_{M} \frac{d \tau}{d \mu}(x) \frac{d \tau_{1}}{d \mu}(x) \frac{d \tau_{2}}{d \mu}(x) d \mu(x)
$$

One obtains similar formulae for the terms $\tau_{\mu} G\left(\tau_{1}, \tau_{2}\right),\left(\tau_{1}\right)_{\mu} G\left(\tau, \tau_{2}\right)$ and then finally

$$
G_{\mu}\left(\nabla_{\tau_{2}} \tau, \tau_{1}\right)=-\frac{1}{2} \int_{M} \frac{d \tau}{d \mu}(x) \frac{d \tau_{2}}{d \mu}(x) \frac{d \tau_{1}}{d \mu}(x) d \mu(x)
$$

On the other hand, one observes

$$
\int_{M} G_{\mu}\left(\tau, \tau_{2}\right) \frac{d \tau_{1}}{d \mu}(x) d \mu(x)=G_{\mu}\left(\tau, \tau_{2}\right) \int_{M} d \tau_{1}(x)=0
$$

and hence

$$
\begin{aligned}
G_{\mu}\left(\nabla_{\tau_{2}} \tau, \tau_{1}\right) & =-\frac{1}{2} \int_{M}\left(\frac{d \tau}{d \mu}(x) \frac{d \tau_{2}}{d \mu}(x)-G_{\mu}\left(\tau, \tau_{2}\right)\right) \frac{d \tau_{1}}{d \mu}(x) d \mu(x) \\
& =G_{\mu}\left(-\frac{1}{2}\left(\frac{d \tau}{d \mu}(x) \frac{d \tau_{2}}{d \mu}(x)-G_{\mu}\left(\tau, \tau_{2}\right)\right) \mu, \tau_{1}\right)
\end{aligned}
$$

Since $\tau_{1}$ is arbitrary, (13) is derived.

Theorem 9. The Riemannian curvature tensor $R$ of the Fisher metric $G$ satisfies

$$
R_{\mu}\left(\tau_{1}, \tau_{2}\right) \tau=\frac{1}{4}\left(G_{\mu}\left(\tau, \tau_{2}\right) \tau_{1}-G_{\mu}\left(\tau, \tau_{1}\right) \tau_{2}\right)
$$

for constant vector fields $\tau, \tau_{1}, \tau_{2}$. Hence, sectional curvature of any section $\tau \wedge \tau_{1}$ is $K\left(\tau \wedge \tau_{1}\right)=\frac{1}{4}$. 
Refer to [2] for this theorem. We omit proving this theorem. In general, a finite dimensional Riemannian manifold of constant sectional curvature $1 / 4$ is (locally) isometric to a sphere of radius 2 . So, the space $\mathcal{P}(M)$ with the metric $G$ is considered to be isometrically an infinite dimensional sphere of radius 2 .

As is shown in the next section, this infinite dimensional Riemannian manifold $(\mathcal{P}(M), G)$ is not geodesically complete, in other words, every geodesic is not necessarily extended over $\mathbf{R}$.

\subsection{Geodesics}

Theorem 10. Let $\mu \in \mathcal{P}(M)$ and $\tau \in T_{\mu} \mathcal{P}(M)$. Assume $\tau$ is a unit tangent vector at $\mu$, i.e., $|\tau|_{G, \mu}=1$. Then, the geodesic $\mu(t)$, denoted by $\exp _{\mu} t \tau$, with $\mu(0)=\mu, \dot{\mu}(0)=\tau$ has the form represented by

$$
\mu(t)=\left(\cos \frac{t}{2}+\sin \frac{t}{2} \frac{d \tau}{d \mu}(x)\right)^{2} \mu
$$

in other words,

$$
\mu(t)=\left(\cos \frac{t}{2}+\sin \frac{t}{2} \frac{q(x)}{p(x)}\right)^{2} p(x) d \theta(x)
$$

where $\mu=p(x) d \theta$ and $\tau=q(x) d \theta$ are density function representation of $\mu, \tau$.

Note. Set $t=\pi$ into (15). Then $\mu(\pi)=\left(\frac{d \tau}{d \mu}(x)\right)^{2} \mu=\frac{q(x)^{2}}{p(x)} d \theta$. However, $\tau$ is a tangent vector to $\mathcal{P}(M)$ so $\tau$ satisfies $\int_{x \in M} q(x) d \theta(x)=0$ from which there exists a point $x_{o} \in M$ with $q\left(x_{o}\right)=0$ and then the density function of $\mu(\pi)$ vanishes at point $x_{o}$ and then $\mu(\pi) \notin \mathcal{P}(M)$.

To prove Theorem 10, we will show the following lemma, obtained by T. Friedrich ([2]).

Lemma 1. Let $\mu(t)=p(x, t) d \theta$ be a geodesic such that $\mu(0)=\mu=p_{0}(x) d \theta, \dot{\mu}(0)=\tau=q(x) d \theta \in$ $T_{\mu} \mathcal{P}^{+}(M),|\tau|_{G, \mu}=1$. Then,

$$
p(x, t)=\frac{1}{1+\tan ^{2} \frac{t}{2}}\left\{p_{0}(x)+2 \tan \frac{t}{2} q(x)+\tan ^{2} \frac{t}{2} \frac{q^{2}(x)}{p_{0}(x)}\right\} .
$$

From this lemma it is easy to see that $\mu(t)=p(x, t) d \theta$ has the above form (15).

Proof of Lemma 1. A proof is given in [2]. However, we will give a proof for a later convenience in proving Theorems 16 and 17. So, for simplicity we write by abbreviation $\mu(t)=p(t) d \theta$ and $\dot{\mu}(t)=$ $\dot{p}(t) d \theta$. Letting $\tau$ be a constant vector field, we have

$$
G\left(\nabla_{\dot{\mu}(t)} \dot{\mu}(t), \tau\right)=\dot{\mu}(t)(G(\dot{\mu}(t), \tau))-G\left(\dot{\mu}(t), \nabla_{\dot{\mu}(t)} \tau\right),
$$

since $\nabla$ preserves the metric $G$.

Notice that from the rule (14) of Levi-Civita connection, the tangent vector $\dot{\mu}(t) \in T_{\mu(t)} \mathcal{P}(M)$ of $\nabla_{\dot{\mu}(t)}$, appeared in the second term of (18) can be extended as a constant vector field, denoted by the same symbol. So, we can apply (13) to the above and have

$$
\nabla_{\dot{\mu}(t)} \tau=-\frac{1}{2}\left(\frac{d \dot{\mu}(t)}{d \mu(t)} \frac{d \tau}{d \mu(t)}-G_{\mu(t)}(\dot{\mu}(t), \tau)\right) \mu(t) .
$$


Thus, the Radon-Nikodym derivative of $\nabla_{\dot{\mu}(t)} \tau$ with respect to $\mu(t)$ is

$$
\frac{d \nabla_{\dot{\mu}(t)} \tau}{d \mu(t)}=-\frac{1}{2}\left(\frac{d \dot{\mu}(t)}{d \mu(t)} \frac{d \tau}{d \mu(t)}-G_{\mu(t)}(\dot{\mu}(t), \tau)\right) .
$$

Therefore, we have

$$
\begin{aligned}
G\left(\dot{\mu}(t), \nabla_{\dot{\mu}(t)} \tau\right) & =\int_{M}\left(\frac{d \dot{\mu}(t)}{d \mu(t)}\right)\left\{-\frac{1}{2}\left(\frac{d \dot{\mu}(t)}{d \mu(t)} \frac{d \tau}{d \mu(t)}-G_{\mu(t)}(\dot{\mu}(t), \tau)\right)\right\} d \mu(t) \\
& =-\frac{1}{2} \int_{M}\left(\frac{d \dot{\mu}(t)}{d \mu(t)}\right)^{2} \frac{d \tau}{d \mu(t)} d \mu(t)+\frac{1}{2} G_{\mu(t)}(\dot{\mu}(t), \tau) \int_{M}\left(\frac{d \dot{\mu}(t)}{d \mu(t)}\right) d \mu(t) \\
& =-\frac{1}{2} \int_{M}\left(\frac{d \dot{\mu}(t)}{d \mu(t)}\right)^{2} d \tau+\frac{1}{2} G_{\mu(t)}(\dot{\mu}(t), \tau) \int_{M} d \dot{\mu}(t) \\
& =-\frac{1}{2} \int_{M}\left(\frac{d \dot{\mu}(t)}{d \mu(t)}\right)^{2} d \tau=-\frac{1}{2} \int_{M}\left(\frac{\dot{p}(t)}{p(t)}\right)^{2} d \tau
\end{aligned}
$$

On the other hand, the first term of (18) is

$$
\dot{\mu}(t) G(\dot{\mu}(t), \tau)=\frac{d}{d t} G_{\mu(t)}(\dot{\mu}(t), \tau)=\frac{d}{d t} \int_{M}\left(\frac{\dot{p}(t)}{p(t)}\right) d \tau=\int_{M} \frac{\partial}{\partial t}\left(\frac{\dot{p}(t)}{p(t)}\right) d \tau .
$$

Then (18) becomes

$$
G\left(\nabla_{\dot{\mu}(t)} \dot{\mu}(t), \tau\right)=\int_{M}\left\{\frac{\partial}{\partial t}\left(\frac{\dot{p}(t)}{p(t)}\right)+\frac{1}{2}\left(\frac{\dot{p}(t)}{p(t)}\right)^{2}\right\} d \tau .
$$

Here, $\left\{\frac{\partial}{\partial t}\left(\frac{\dot{p}(t)}{p(t)}\right)+\frac{1}{2}\left(\frac{\dot{p}(t)}{p(t)}\right)^{2}\right\} \mu(t)$ is not necessarily a tangent vector at $\mu(t)$. We choose a real valued function $C(t)$ of $t$, independent of $x \in M$ satisfying

$$
\left\{\frac{\partial}{\partial t}\left(\frac{\dot{p}(t)}{p(t)}\right)+\frac{1}{2}\left(\frac{\dot{p}(t)}{p(t)}\right)^{2}+C(t)\right\} \mu(t) \in T_{\mu(t)} \mathcal{P}^{+}(M) .
$$

In fact, we define $C(t)$ as

$$
\begin{aligned}
C(t) & =-\int_{M}\left\{\frac{\partial}{\partial t}\left(\frac{\dot{p}(t)}{p(t)}\right)+\frac{1}{2}\left(\frac{\dot{p}(t)}{p(t)}\right)^{2}\right\} p(t) d \theta \\
& =-\int_{M} \frac{\partial}{\partial t}\left(\frac{d \dot{\mu}(t)}{d \mu(t)}\right) d \mu(t)-\frac{1}{2} G(\dot{\mu}(t), \dot{\mu}(t)) .
\end{aligned}
$$

Hence, we have

$$
G\left(\nabla_{\dot{\mu}(t)} \dot{\mu}(t), \tau\right)=G\left(\left\{\frac{\partial}{\partial t}\left(\frac{\dot{p}(t)}{p(t)}\right)+\frac{1}{2}\left(\frac{\dot{p}(t)}{p(t)}\right)^{2}+C(t)\right\} \mu(t), \tau\right)
$$

for an arbitrary constant vector field $\tau$.

Therefore we have

$$
\nabla_{\dot{\mu}(t)} \dot{\mu}(t)=\left\{\frac{\partial}{\partial t}\left(\frac{\dot{p}(t)}{p(t)}\right)+\frac{1}{2}\left(\frac{\dot{p}(t)}{p(t)}\right)^{2}+C(t)\right\} \mu(t)
$$

Thus, it is concluded that $\mu(t)=p(t) d \theta$ is a geodesic if and only if $p(t)=p(x, t)$ satisfies

$$
\frac{\partial}{\partial t}\left(\frac{\dot{p}(t)}{p(t)}\right)+\frac{1}{2}\left(\frac{\dot{p}(t)}{p(t)}\right)^{2}+C(t)=0, \quad \int_{M} \dot{p}(t) d \theta=0
$$


Remark 2. Equation (19) is expressed as

$$
\frac{\partial}{\partial t}\left(\frac{\dot{\mu}(t)}{\mu(t)}\right)+\frac{1}{2}\left(\frac{\dot{\mu}(t)}{\mu(t)}\right)^{2}+C(t)=0, \quad \dot{\mu}(t) \in T_{\mu(t)} \mathcal{P}(M) .
$$

Now we will solve these equations.

Notice that if $\mu(t)$ is a geodesic, $G_{\mu(t)}(\dot{\mu}(t), \dot{\mu}(t))$ is constant along $\mu(t)$ so from the initial condition $G_{\mu(t)}(\dot{\mu}(t), \dot{\mu}(t)) \equiv 1$. Therefore, we can write (19) as

$$
\begin{gathered}
\frac{\partial}{\partial t}\left(\frac{\dot{p}(t)}{p(t)}\right)+\frac{1}{2}\left(\frac{\dot{p}(t)}{p(t)}\right)^{2}-\int_{M} \frac{\partial}{\partial t}\left(\frac{\dot{p}(t)}{p(t)}\right) p(t) d \theta-\frac{1}{2}=0, \\
\int_{M} \frac{\dot{p}^{2}(t)}{p(t)} d \theta=1, \quad \int_{M} \dot{p}(t) d \theta=0 .
\end{gathered}
$$

The second equation means that $|\dot{\mu}(t)|_{G, \mu(t)}=1$. Set $g(t):=\int_{M} \frac{\partial}{\partial t}\left(\frac{\dot{p}(t)}{p(t)}\right) p(t) d \theta$. We have then

$$
\begin{aligned}
g(t) & =\frac{d}{d t}\left(\int_{M}\left(\frac{\dot{p}(t)}{p(t)}\right) p(t) d \theta\right)-\int_{M}\left(\frac{\dot{p}(t)}{p(t)}\right) \dot{p}(t) d \theta \\
& =\frac{d}{d t}\left(\int_{M} d \dot{\mu}(t)\right)-G_{\mu(t)}(\dot{\mu}(t), \dot{\mu}(t))=0-1=-1 .
\end{aligned}
$$

So Equations (20) reduce to

$$
\frac{\partial}{\partial t}\left(\frac{\dot{p}(t)}{p(t)}\right)+\frac{1}{2}\left(\frac{\dot{p}(t)}{p(t)}\right)^{2}+\frac{1}{2}=0, \quad \int_{M}\left(\frac{\dot{p}(t)}{p(t)}\right)^{2} p(t) d \theta=1, \quad \int_{M} \dot{p}(t) d \theta=0 .
$$

To solve these, we set $w(t)=\frac{\dot{p}(t)}{p(t)}$. Then $w(t)$ satisfies

$$
\dot{w}(t)+\frac{1}{2}\left(w^{2}(t)+1\right)=0 .
$$

By solving (21), we have $w(t)=\tan \left(-\frac{1}{2} t+A(x)\right), x \in M$, where $A(x)$ is an integral constant depending on $x$. By integrating $w(t)=\dot{p}(t) / p(t)$, where $p(t)=p(x, t)$,

$$
\log p(x, t)=2 \log \cos \left(-\frac{1}{2} t+A(x)\right)+B_{1}(x)
$$

and hence

$$
p(x, t)=B(x) \cos ^{2}\left(-\frac{1}{2} t+A(x)\right) .
$$

Here $B(x)$ is an integral constant. The constants $A(x), B(x)$ are given as

$$
\begin{aligned}
& A(x)=\arctan \left(\frac{\dot{p}(x, 0)}{p(x, 0)}\right), \\
& B(x)=\frac{p^{2}(x, 0)+\dot{p}^{2}(x, 0)}{p(x, 0)},
\end{aligned}
$$

where $p(x, 0)=p_{0}(x), \dot{p}(x, 0)=q(x)$.

Corollary 2 ([2]). Every geodesic on $(\mathcal{P}(M), G)$ is periodic, of period $2 \pi$. 
In fact, from (15) a geodesic $\mu(t)$ is represented as

$$
\mu(t)=\left(\cos ^{2} \frac{t}{2}+2 \sin \frac{t}{2} \cos \frac{t}{2} \frac{d \tau}{d \mu}(x)+\sin ^{2} \frac{t}{2}\left(\frac{d \tau}{d \mu}\right)^{2}(x)\right) \mu
$$

and $\cos ^{2} \frac{t}{2}=\frac{1}{2}(1+\cos t)$. We have then the corollary.

Definition 2. Define $\ell: \mathcal{P}(M) \times \mathcal{P}(M) \rightarrow[0, \pi) ;\left(\mu, \mu^{*}\right) \mapsto \ell=\ell\left(\mu, \mu^{*}\right)$ by

$$
\cos \frac{\ell}{2}=\int_{x \in M} \sqrt{\frac{d \mu^{*}}{d \mu}}(x) d \mu(x) .
$$

One sees $\ell\left(\mu, \mu^{*}\right)=\ell\left(\mu^{*}, \mu\right)$ and that $\ell=0$ if and only if $\mu=\mu^{*} \cdot \cos \frac{\ell}{2}$ is an $f$-divergence-like quantity with respect to $f(u)=\sqrt{u}$ (See [14]).

Remark 3. In [2] T. Friedrich remarked that the distance between $\mu$ and $\mu^{*}$ in $\mathcal{P}(M)$ is given by $\ell=$ $\ell\left(\mu, \mu^{*}\right)$.

Theorem 11. Let $\mu$ and $\mu^{*}$ be arbitrary probability measures in $\mathcal{P}(M), \mu \neq \mu^{*}$. Then, there exists a unique geodesic $\mu(t)$, i.e., a curve; $t \in I \subset \mathbf{R} \mapsto \mu(t) \in \mathcal{P}(M)$ with $\mu(0)=\mu, \mu(\ell)=\mu^{*}$, where $\ell=\ell\left(\mu, \mu^{*}\right)$ is given by (22) and I is an open interval;

$$
\mu(t)=\exp _{\mu} t \tau=\left(\cos \frac{t}{2}+\sin \frac{t}{2} \frac{d \tau}{d \mu}(x)\right)^{2} \mu,
$$

where $\tau$ is a unit tangent vector at $\mu$ represented by

$$
\tau=\frac{1}{\sin \frac{\ell}{2}}\left(\sqrt{\frac{d \mu^{*}}{d \mu}}(x)-\int_{y \in M} \sqrt{\frac{d \mu^{*}}{d \mu}}(y) d \mu(y)\right) \mu(x) .
$$

Proof. First we will show

Assertion 1. The measure $\tau$ given by (24) is a unit tangent vector to $\mathcal{P}(M)$ at $\mu$.

In fact,

$$
\begin{aligned}
\int_{M} d \tau & =\frac{1}{\sin \frac{\ell}{2}} \int_{x \in M}\left(\sqrt{\frac{d \mu^{*}}{d \mu}}(x)-\int_{y \in M} \sqrt{\frac{d \mu^{*}}{d \mu}}(y) d \mu(y)\right) d \mu(x) \\
& =\frac{1}{\sin \frac{\ell}{2}}\left(\int_{x \in M} \sqrt{\frac{d \mu^{*}}{d \mu}}(x) d \mu(x)-\int_{y \in M} \sqrt{\frac{d \mu^{*}}{d \mu}}(y) d \mu(y)\right)=0,
\end{aligned}
$$

so that $\tau$ is a tangent vector to $\mathcal{P}(M)$. Moreover, $\tau$ is unit, i.e., $G_{\mu}(\tau, \tau)=1$, as we compute straightforward

$$
G_{\mu}(\tau, \tau)=\int_{M}\left(\frac{d \tau}{d \mu}(x)\right)^{2} d \mu(x)
$$


and substitute (24) into (25) to have

$$
\begin{aligned}
G_{\mu}(\tau, \tau)= & \frac{1}{\sin ^{2} \frac{\ell}{2}} \int_{x \in M}\left(\sqrt{\frac{d \mu^{*}}{d \mu}}(x)-\int_{y \in M} \sqrt{\frac{d \mu^{*}}{d \mu}}(y) d \mu(y)\right)^{2} d \mu(x) \\
= & \frac{1}{\sin ^{2} \frac{\ell}{2}} \int_{x \in M}\left\{\frac{d \mu^{*}}{d \mu}(x)-2\left(\int_{y \in M} \sqrt{\frac{d \mu^{*}}{d \mu}}(y) d \mu(y)\right) \sqrt{\frac{d \mu^{*}}{d \mu}}(x)\right. \\
& \left.+\left(\int_{y \in M} \sqrt{\frac{d \mu^{*}}{d \mu}}(y) d \mu(y)\right)^{2}\right\} d \mu(x) \\
= & \frac{1}{\sin ^{2} \frac{\ell}{2}}\left\{\int_{x \in M} d \mu^{*}(x)-2\left(\int_{y \in M} \sqrt{\frac{d \mu^{*}}{d \mu}}(y) d \mu(y)\right)^{2}+\left(\int_{y \in M} \sqrt{\frac{d \mu^{*}}{d \mu}}(y) d \mu(y)\right)^{2}\right\} \\
= & \frac{1}{\sin ^{2} \frac{\ell}{2}}\left(1-\cos ^{2} \frac{\ell}{2}\right)=1 .
\end{aligned}
$$

Assertion 2. A geodesic defined by (23) satisfies $\mu(0)=\mu$ and $\mu(\ell)=\mu^{*}$.

It is seen $\mu(0)=\mu$ from (23). At $t=\ell$

$$
\mu(\ell)=\left(\cos \frac{\ell}{2}+\sin \frac{\ell}{2} \frac{d \tau}{d \mu}(x)\right)^{2} \mu
$$

and from (25)

$$
\sin \frac{\ell}{2} \frac{d \tau}{d \mu}(x)=\sqrt{\frac{d \mu^{*}}{d \mu}}(x)-\int_{y \in M} \sqrt{\frac{d \mu^{*}}{d \mu}}(y) d \mu(y)=\sqrt{\frac{d \mu^{*}}{d \mu}}(x)-\cos \frac{\ell}{2} .
$$

Hence, we find $\mu(\ell)=\mu^{*}$ as follows;

$$
\mu(\ell)=\left\{\cos \frac{\ell}{2}+\left(\sqrt{\frac{d \mu^{*}}{d \mu}}(x)-\cos \frac{\ell}{2}\right)\right\}^{2} \mu=\left(\sqrt{\frac{d \mu^{*}}{d \mu}}(x)\right)^{2} \mu=\frac{d \mu^{*}}{d \mu}(x) \mu=\mu^{*} .
$$

Assertion 3. A geodesic joining $\mu$ and $\mu^{*}$ is unique for $\mu \neq \mu^{*}$.

To verify this assertion let $\mu(t)=\exp _{\mu} t \tau$ and $\tilde{\mu}(t)=\exp _{\mu} t \tilde{\tau}$ be unit speed geodesics satisfying $\mu(0)=\tilde{\mu}(0)=\mu$ and $\mu(\ell)=\tilde{\mu}(\ell)=\mu^{*}$. From the latter condition we have by using (15)

$$
\left(\cos \frac{\ell}{2}+\sin \frac{\ell}{2} \frac{d \tilde{\tau}}{d \mu}(x)\right)^{2} \mu=\left(\cos \frac{\ell}{2}+\sin \frac{\ell}{2} \frac{d \tau}{d \mu}(x)\right)^{2} \mu, \quad \forall x \in M
$$

from which

$$
\cos \frac{\ell}{2}+\sin \frac{\ell}{2} \frac{d \tilde{\tau}}{d \mu}(x)= \pm\left(\cos \frac{\ell}{2}+\sin \frac{\ell}{2} \frac{d \tau}{d \mu}(x)\right)
$$

for any $x \in M$.

To assert

$$
\cos \frac{\ell}{2}+\sin \frac{\ell}{2} \frac{d \tilde{\tau}}{d \mu}(x)=\cos \frac{\ell}{2}+\sin \frac{\ell}{2} \frac{d \tau}{d \mu}(x), \quad \forall x \in M
$$

define subsets $M_{ \pm}$of $M$ by

$$
M_{ \pm}=\left\{x \in M ; \cos \frac{\ell}{2}+\sin \frac{\ell}{2} \frac{d \tilde{\tau}}{d \mu}(x)= \pm\left(\cos \frac{\ell}{2}+\sin \frac{\ell}{2} \frac{d \tau}{d \mu}(x)\right)\right\} .
$$


Then, $M=M_{+} \cup M_{-}$. Moreover $M_{+} \cap M_{-}=\emptyset$. This is because if otherwise, $M_{+} \cap M_{-} \neq \emptyset$, then at a point $x \in M_{+} \cap M_{-}$it holds

$$
\cos \frac{\ell}{2}+\sin \frac{\ell}{2} \frac{d \tilde{\tau}}{d \mu}(x)=0
$$

So, at $x, \tilde{\mu}(\ell)=\left(\cos \frac{\ell}{2}+\sin \frac{\ell}{2} \frac{d \tilde{\tau}}{d \mu}(x)\right)^{2} \mu=0$. However, it must coincide at $x$ with $\mu^{*}$ which has a positive density function. So $M_{+} \cap M_{-}=\emptyset$.

We see that $M_{+}=\{x \in M ; \tilde{\tau}(x)=\tau(x)\}$ and $M_{-}=\left\{x \in M ; q(x)+\tilde{q}(x)=-2 \cot \frac{\ell}{2}\right\}$. Here we write $\tau=q(x) d \theta$ and $\tilde{\tau}=\tilde{q}(x) d \theta$. Then, $M_{+}$and $M_{-}$are closed in $M$.

Suppose $M_{-} \neq \emptyset$. Then, $M_{-}$must be a non-empty, proper subset of $M$. This is because, otherwise if $M_{-}=M$ is assumed, then, since $\tilde{\tau}, \tau$ are tangent to $\mathcal{P}(M)$ we see, from $0<\ell<\pi$

$$
\int_{M} d \tilde{\tau}=-\int_{M} d \tau-2 \cot \frac{\ell}{2} \int_{M} d \theta=-2 \cot \frac{\ell}{2} \neq 0 .
$$

This is a contradiction. So, $M_{-}$is a proper subset and hence $M_{+}=M \backslash M_{-}$is a non-empty closed, but open subset of $M$. Therefore, since $M$ is connected, $M_{+}=M$, namely $\tilde{\tau}(x)=\tau(x)$ for any $x \in X$, from which the assertion is proved.

From these assertions Theorem 11 is verified.

Remark 4. For the $\ell$ of (22)

$$
\mu^{*} \neq \mu \Longleftrightarrow \sin \frac{\ell}{2} \neq 0 .
$$

It suffices for this to show

$$
\mu^{*}=\mu \Longleftrightarrow \ell=0,
$$

since, for $\ell \in[0, \pi), \sin \ell / 2=0$ if and only if $\ell=0$. With respect to the embedding $\rho: \mathcal{P}(M) \rightarrow$ $L^{2}(M, d \theta)$, given in (8), we have

$$
\left\|\rho\left(\mu^{*}\right)-\rho(\mu)\right\|_{L^{2}}^{2}=2-2 \cos \frac{\ell}{2}
$$

from which it follows that $\ell=0$ implies $\left\|\rho\left(\mu^{*}\right)-\rho(\mu)\right\|_{L^{2}}=0$ and hence $\mu^{*}=\mu$, since $\rho$ is an embedding. Conversely, if $\mu^{*}=\mu$, then $\sqrt{d \mu^{*} / d \mu}(x)=1$ so $\cos \ell / 2=\int_{M} \sqrt{d \mu^{*} / d \mu}(x) d \mu=1$ and thus $\ell=0$.

To guarantee completeness of geodesics we must extend the space $\mathcal{P}(M)$, for example, to the space of probability measures on $M$, absolutely continuous with respect to $d \theta$ and with non-negative density function.

\section{Hadamard Manifolds and Barycenter Map}

\subsection{Hadamard Manifolds and Ideal Boundary}

Let $(X, g)$ be an Hadamard manifold. Then the ideal boundary $\partial X$ of $(X, g)$ is defined by taking quotient of space of geodesics of $X$ and is homeomorphic to an $(n-1)$-sphere $S^{n-1}$. For any $\theta \in \partial X$ Busemann function $B_{\theta}$ normalized at some point and parametrized in $\theta \in \partial X$ provides a $\mu$-average 
Busemann function $\mathbf{B}_{\mu}$ on $X$ in terms of a probability measure $\mu$ on $\partial X$. Under some geometrical assumptions which $X$ fulfills, $\mathbf{B}_{\mu}$ admits a unique critical point so that we have a barycenter map bar : $\mathcal{P}(\partial X) \rightarrow X$ by assigning to an arbitrary probability measure $\mu$ on $\partial X$ a point in $X$ as its barycenter, a critical point of $\mathbf{B}_{\mu}$.

Let $(X, g)$ be an Hadamard manifold, a simply connected, complete Riemannian manifold with a metric $g=\langle\cdot, \cdot\rangle$ of non-positive curvature. By Cartan-Hadamard theorem an Hadamard manifold is diffeomorphic to a Euclidean space of same dimension. Refer, for this theorem, to [30,31]

A Euclidean space together with a real hyperbolic space $H^{n}(\mathbf{R})$ are typical examples of Hadamard manifold. Geometrical properties which an Hadamard manifold enjoys are the following;

(i) Any two points on $X$ can be joined by a unique geodesic.

(ii) Let $\Delta$ be a geodesic triangle in $X$ with interior angles $\alpha_{1}, \alpha_{2}, \alpha_{3}$ and lengths of opposite side, $\ell_{1}$, $\ell_{2}, \ell_{3}$. Then, we have a law of cosines;

$$
\ell_{3}^{2} \geq \ell_{1}^{2}+\ell_{2}^{2}-2 \ell_{1} \ell_{2} \cos \alpha_{3}
$$

(iii) The distance function from a fixed point $x_{o} \in X ; d_{x_{o}}: X \rightarrow \mathbf{R}, x \mapsto d\left(x, x_{o}\right)$ is convex, i.e., for any geodesic $\gamma$ in $X ; t \mapsto \gamma(t)$ the restricted function $d_{x_{o}} \circ \gamma: t \mapsto d_{x_{o}}(\gamma(t))$ is convex on $\mathbf{R}$.

Here a function $f: \mathbf{R} \rightarrow \mathbf{R}$ is convex, if it satisfies

$$
f\left(a t_{1}+(1-a) t_{2}\right) \leq a f\left(t_{1}\right)+(1-a) f\left(t_{2}\right), \quad \forall t_{1}, t_{2} \in \mathbf{R}, 0 \leq a \leq 1 .
$$

Let us define for an Hadamard manifold $(X, g)$ the ideal boundary $\partial X$, or a boundary at infinity.

Let $\gamma, \sigma ; \mathbf{R} \rightarrow X$ be unit speed geodesics on $X$. We say that $\gamma$ is asymptotically equivalent with $\sigma$, denoted by $\gamma \sim \sigma$, when there exists a constant $C>0$ such that $d(\gamma(t), \sigma(t)) \leq C$ for any $t \geq 0$. The relation $\sim$ is an equivalence relation on the space $\operatorname{Geo}(X)$ of all oriented, unit speed geodesics on $X$. The quotient space $\operatorname{Geo}(X) / \sim$ is called the ideal boundary of $X$, denoted by $\partial X$. An equivalence class represented by $\gamma \in \operatorname{Geo}(X)$ is called an asymptotic class, denoted by $[\gamma]$ or $\gamma(\infty)$. Notice that all geodesics on $X$ are assumed to be of unit speed and oriented.

Let $x \in X$ be an arbitrary point of $X$ and $S_{x} X$ the space of unit tangent vectors at $x$;

$$
S_{x} X=\left\{v \in T_{x} X ;\|v\|=1\right\}
$$

Then we define a map

$$
\beta=\beta_{x}: S_{x} X \rightarrow \partial X ; \quad v \mapsto[\gamma]
$$

where $\gamma \in \operatorname{Geo}(X)$ is a geodesic given by $\gamma(t)=\exp _{x}(t v), t \in \mathbf{R}$.

Proposition 1 ([30]). The map $\beta_{x}$ is bijective.

Moreover, we equip the space $X \cup \partial X$ with a topology, called a cone topology as follows. For $x \in X$ and $\theta_{1}, \theta_{2} \in X \cup \partial X\left(x \neq \theta_{1}, x \neq \theta_{2}\right)$ we define $L_{x}\left(\theta_{1}, \theta_{2}\right)=\angle\left(\dot{\gamma}_{1}(0), \dot{\gamma}_{2}(0)\right)$ angles between a geodesic $\gamma_{1}$ from $x$ to $\theta_{1}$ and a geodesic $\gamma_{2}$ from $x$ to $\theta_{2}$. For $x \in X$ and $\theta \in \partial X, \varepsilon>0$ let 
$C_{x}(\theta, \varepsilon)=\left\{\theta_{1} \in X \cup \partial X ; \theta_{1} \neq x, L_{x}\left(\theta, \theta_{1}\right)<\varepsilon\right\}$ be a cone. Further, let $T_{x}(\theta, \varepsilon)=C_{x}(\theta, \varepsilon) \backslash B(x, r)$ be a truncated cone $(B(x, r)=\{y \in X \mid d(y, x) \leq r\}$ is a closed geodesic ball). Then, a topology generated by open geodesic balls in $X$ and such truncated cones is called a cone topology of $X \cup \partial X$. Notice that thus defined cone topology when restricted to $\partial X$ is homeomorphic to the usual topology on $S_{x} X$ via the mapping $\beta_{x}$. Refer to [31] for the detail.

Let $(d \theta)_{x}$ be a standard volume measure on $S_{x} X$, normalized by $(d \theta)_{x}\left(S_{x} X\right)=1$. Through $\beta_{x}$ we obtain a measure $\left(\beta_{x}\right)_{\sharp}(d \theta)_{x}$ on $\partial X$, denoted by $d \theta$.

\subsection{Normalized Busemann Function}

Busemann function on $X$ is introduced to define an average Busemann function in terms of a probability measure on $\partial X$.

Let $\gamma: \mathbf{R} \rightarrow X$ be a geodesic on $X$. Define a function $f_{t}: X \rightarrow \mathbf{R}$ for $t>0$ by

$$
f_{t}(x)=d_{x}(\gamma(t))-t=d(x, \gamma(t))-d(\gamma(0), \gamma(t))
$$

For any $x \in X$ a limit $\lim _{t \rightarrow \infty} f_{t}(x)$ exists, as we will see in Proposition 2. We write this limit as $f_{\infty}(x)$ and define a function on $X ; x \mapsto f_{\infty}(x)$, called Busemann function, denoted by $B_{\gamma}: X \rightarrow \mathbf{R}$; $x \mapsto f_{\infty}(x)$.

Each level set of $B_{\gamma}$ is called a horosphere, important in studying geometry of Hadamard manifolds. See [11], for example.

Example 1. In a Euclidean space $(X, g)=\left(\mathbf{R}^{n}, g_{o}\right)$ let $\gamma$ be a geodesic, $\gamma(t)=(t, 0, \ldots, 0)$. Then, $B_{\gamma}(\mathbf{x})=-x^{1}$ for $\mathbf{x}=\left(x^{1}, \ldots, x^{n}\right) \in \mathbf{R}^{n}$ from the following

$$
f_{t}(\mathbf{x})=d(\mathbf{x}, \gamma(t))-t=\sqrt{\left(x^{1}-t\right)^{2}+\sum_{i=2}^{n}\left(x^{i}\right)^{2}}-t=\frac{-2 t x^{1}+\sum_{i=2}^{n}\left(x^{i}\right)^{2}}{\sqrt{\left(x^{1}-t\right)^{2}+\sum_{i=2}^{n}\left(x^{i}\right)^{2}}+t} \rightarrow-x^{1}
$$

as $t \rightarrow \infty$.

Example 2. Busemann function on $H^{n}(\mathbf{R})$, an n-dimensional real hyperbolic space with standard hyperbolic metric, normalized at o, is given

$$
B_{\gamma}(x)=-\log \frac{1-|x|^{2}}{|x-\theta|^{2}}
$$

where $\theta=\gamma(\infty) \in S^{n-1}$.

Proposition 2. The functions $f_{t}: X \rightarrow \mathbf{R}, t>0$, introduced above, have a limit $\lim _{t \rightarrow \infty} f_{t}(x)$ for each $x \in X$.

From the triangle inequality we have

$$
t_{1}<t_{2} \Longrightarrow f_{t_{1}}(x) \geq f_{t_{2}}(x), \quad \forall x \in X .
$$

In fact, since $d\left(\gamma\left(t_{1}\right), \gamma\left(t_{2}\right)\right)=t_{2}-t_{1}$, we see

$$
d\left(x, \gamma\left(t_{2}\right)\right) \leq d\left(x, \gamma\left(t_{1}\right)\right)+d\left(\gamma\left(t_{1}\right), \gamma\left(t_{2}\right)\right)=d\left(x, \gamma\left(t_{1}\right)\right)+\left(t_{2}-t_{1}\right)
$$


from which the above is derived. On the other hand, we observe uniform boundedness of $f_{t}$, that is $\left|f_{t}(x)\right| \leq d(\gamma(0), x)$ for any $x \in X$ and $t>0$ as follows;

$$
f_{t}(x)=d(x, \gamma(t))-t \leq d(x, \gamma(0))+d(\gamma(0), \gamma(t))-t=d(x, \gamma(0))
$$

and

$$
\begin{aligned}
t-d(x, \gamma(t)) & =d(\gamma(0), \gamma(t))-d(x, \gamma(t)) \\
& \leq d(\gamma(0), x)+d(x, \gamma(t))-d(x, \gamma(t))=d(x, \gamma(0))
\end{aligned}
$$

so $-d(x, \gamma(0)) \leq f_{t}(x) \leq d(x, \gamma(0))$.

Therefore, the sequence $\left\{f_{t}(x) \mid t>0\right\}$ is bounded and decreasing and then has a limit as $t \rightarrow \infty$.

Proposition 3. Let $\gamma$ and $\sigma$ be geodesics. If $\gamma \sim \sigma$, then

$$
B_{\gamma}(x)-B_{\sigma}(x)=c, \quad \forall x \in X
$$

for a constant $c$.

See [30,31] for this proposition, from which, for $\theta \in \partial X$ Busemann function $B_{\gamma}$ associated with a geodesic $\gamma,[\gamma]=\theta$, gives us a same function on $X$ modulo additive constant. So, let $x_{o} \in X$ be an arbitrary point of $X$ as a base point. Then, from non-positive curvature of $X$ there exists a unique geodesic $\gamma$ such that $\gamma(0)=x_{o}$ and $[\gamma]=\theta$.

Definition 3. Let $x_{o} \in X$ and $\theta \in \partial X$. Let $\gamma: \mathbf{R} \rightarrow X$ be a geodesic satisfying $\gamma(0)=x_{o}$ and $[\gamma]=\theta$. The Busemann function $B_{\gamma}$ associated to $\gamma$ is called normalized Busemann function, denoted by $B_{\theta}$.

\section{Properties of (normalized) Busemann function:}

(i) $B_{\theta}\left(x_{o}\right)=0$ for any $\theta \in \partial X$,

(ii) $B_{\theta}(\gamma(t))=-t, t \in \mathbf{R}$, for any $\theta \in \partial X$, where $\gamma$ is a geodesic satisfying $\gamma(0)=x_{o},[\gamma]=\theta$.

(iii) Busemann function is Lipschitz continuous;

$$
\left|B_{\theta}(x)-B_{\theta}(y)\right| \leq d(x, y), \quad x, y \in X
$$

(iv) Busemann function is of class $C^{2}$ (refer to [32]).

(v) Gradient vector field $\nabla B_{\theta}$ satisfies $\left|\left(\nabla B_{\theta}\right)_{x}\right| \equiv 1$ for any $x \in X$ and $\theta \in \partial X$. Here $\left(\nabla B_{\theta}\right)_{x} \in$ $T_{x} X$ is defined by $\left\langle\left(\nabla B_{\theta}\right)_{x}, v\right\rangle=v\left(B_{\theta}\right)$, directional derivative of $B_{\theta}$ with respect to $v \in T_{x} X$. An integral curve $x(t)$ of $\nabla B_{\theta}$ passing through a point $x$ is obtained by $x(t)=\sigma(-t)$, where $\sigma$ is a geodesic of $\sigma(0)=x$ and $[\sigma]=\theta$. Moreover, for any $x \in X$ and any vector $v \in S_{x} X$ there exists a $\theta \in \partial X$ such that $v=-\left(\nabla B_{\theta}\right)_{x}$ so that $\beta_{x}(v)=\theta$.

(vi) Busemann function is convex (see (26)), since it is a limit of convex functions. 
(vii) From (vi), the Hessian of Busemann function $\left(\nabla d B_{\theta}\right)_{x}: T_{x} \times T_{x} \rightarrow \mathbf{R}$ is positive semi-definite at any point $x \in X$, i.e., $\left(\nabla d B_{\theta}\right)_{x}(v, v) \geq 0$, for any $v \in T_{x} X$ and $x \in X$, and satisfies

$$
\left(\nabla d B_{\theta}\right)_{x}\left(\left(\nabla B_{\theta}\right)_{x}, v\right)=0, \quad v \in T_{x} X .
$$

Here, for a $C^{2}$-function $f$ on $X$ the Hessian $\nabla d f$ is a symmetric bilinear form, defined by

$$
(\nabla d f)_{x}(u, v)=u(V f)-\left(\nabla_{u} V\right) f, \quad u, v \in T_{x} X, x \in X
$$

where $V$ is a smooth vector field, an extension of $v$. Notice that for a unit vector $u \in S_{x} X$

$$
(\nabla d f)_{x}(u, u)=\left.\frac{d^{2}}{d t^{2}}\right|_{t=0} f(\gamma(t))
$$

with respect to a geodesic $\gamma$ such that $\gamma(0)=x, \dot{\gamma}(0)=u$.

Example 3. On a Euclidean space $\nabla d B_{\theta}=0$ for any $\theta \in \partial X$ (due to Example 1).

Example 4. On a real hyperbolic space $H^{n}(\mathbf{R}), n \geq 2$,

$$
\left(\nabla d B_{\theta}\right)_{x}(u, v)=\langle u, v\rangle-\left\langle\nabla B_{\theta}, u\right\rangle\left\langle\nabla B_{\theta}, u\right\rangle, \quad u, v \in T_{x} X
$$

See for this [8].

Let $(X, g)$ be an Hadamard manifold and $\phi: X \rightarrow X$ be an isometry of $X$, i.e., a smooth transformation of $X$ satisfying $\phi^{*} g=g$. An isometry preserves the distance $d$ of $X$, i.e., $d(\phi(x), \phi(y))=$ $d(x, y), x, y \in X$ and transforms a geodesic $\sigma$ into a new geodesic $\phi \circ \sigma$ so that, if $\sigma \sim \gamma$, then $\phi \circ \sigma \sim \phi \circ \gamma$. Therefore, $\phi$ induces a transformation $\hat{\phi}$ of $\partial X$, a $\partial X$-extension of $\phi$ as

$$
\hat{\phi}: \partial X \rightarrow \partial X ; \quad \theta=[\gamma] \mapsto[\phi \circ \gamma]
$$

Notice that $(\hat{\phi})^{-1}=\widehat{\phi^{-1}}$ for the inverse $\phi^{-1}$ of $\phi . \hat{\phi}$ is a homeomorphism of $\partial X$ in terms of cone topology.

Proposition 4 (Busemann cocycle formula [33]). Any normalized Busemann function enjoys a cocycle formula with respect to an isometry $\phi$ of $X$;

$$
B_{\theta}(\phi(x))=B_{\hat{\phi}^{-1}(\theta)}(x)+B_{\theta}\left(\phi\left(x_{o}\right)\right) .
$$

Proof. Let $\gamma: \mathbf{R} \rightarrow X$ be a geodesic, $\gamma(0)=x_{o},[\gamma]=\theta$. Notice that $\phi \circ \gamma$ is a geodesic with $\phi \circ \gamma(0)=\phi\left(x_{o}\right)$, which, in general, does not coincide with the base point $x_{o}$. For the Busemann function $B_{\phi^{-1} \circ \gamma}(x)$ with respect to a geodesic $\phi^{-1} \circ \gamma$ we have

$$
\begin{aligned}
B_{\phi^{-1} \circ \gamma}(x) & =\lim _{t \rightarrow \infty}\left(d\left(x, \phi^{-1} \circ \gamma(t)\right)-t\right) \\
& =\lim _{t \rightarrow \infty}(d(\phi(x), \gamma(t))-t)=B_{\gamma}(\phi(x)) .
\end{aligned}
$$

On the other hand, $\phi^{-1} \circ \gamma$ belongs to $\widehat{\phi}^{-1}(\theta)$ and $\left(\phi^{-1} \circ \gamma\right)(0)=\phi^{-1}\left(x_{o}\right)$. Let $\sigma$ be a geodesic such that $[\sigma]=\widehat{\phi}^{-1}(\theta), \sigma(0)=x_{o}$. Then, the normalized Busemann function $B_{\widehat{\phi}^{-1}(\theta)}$ is given by $B_{\sigma}$. Since 
$\phi^{-1} \circ \gamma$ and $\sigma$ belong to the same $\widehat{\phi}^{-1}(\theta)$, from (29) $B_{\sigma}-B_{\phi^{-1} \circ \gamma}$ is a constant function on $X$. This constant is given from the above by $\left(B_{\sigma}-B_{\phi^{-1} \circ \gamma}\right)\left(x_{o}\right)=-B_{\phi^{-1} \circ \gamma}\left(x_{o}\right)=-B_{\gamma}\left(\phi\left(x_{o}\right)\right)$ so that on $X$

$$
B_{\sigma}(x)-B_{\phi^{-1} \circ \gamma}(x) \equiv-B_{\gamma}\left(\phi\left(x_{o}\right)\right)
$$

Since $B_{\theta}$ is given by $B_{\gamma}$, (28) is obtained from (30).

In what follows, every normalized Busemann function $B_{\theta}$ on $X$ is assumed to be continuous with respect to $\theta \in \partial X$ for each fixed point $x \in X$. This assumption is guaranteed by a real hyperbolic space. See Example 3. Rank one symmetric spaces of non-compact type and Damek-Ricci spaces satisfy this assumption, as is seen in [25].

Definition 4 ([21]). An Hadamard manifold $(X, g)$ is said to satisfy visibility axiom, if for any distinct ideal point $\theta, \theta_{1}$ of $\partial X$ there exists a geodesic $\gamma: \mathbf{R} \rightarrow X$ such that $\gamma(+\infty)=\theta$ and $\gamma(-\infty)=\theta_{1}$. Here $\gamma(-\infty) \in \partial X$ defined by $\left[\gamma^{-}\right]$, where $\gamma^{-}$is the geodesic of reversed orientation given by $\gamma^{-}(t)=\gamma(-t)$, $t \in \mathbf{R}$.

A Euclidean space does not satisfy visibility axiom.

Proposition 5. Let $(X, g)$ be an Hadamard manifold. $(X, g)$ satisfies visibility axiom if and only if, for any $\theta \in \partial X$

$$
\lim _{x \rightarrow \theta_{1}} B_{\theta}(x)=+\infty
$$

provided $\theta_{1} \neq \theta$. Refer to [31] for this.

Notice $B_{\theta}(x)=-\infty$, if $x \rightarrow \theta$, from property (i) of normalized Busemann function.

\subsection{Average Busemann Function and Barycenter}

In what follows, an Hadamard manifold satisfies visibility axiom and Busemann function $B_{\theta}(x)$ is continuous with respect to every $\theta$.

Let $\partial X$ be, as before, the ideal boundary of an Hadamard manifold $(X, g)$, diffeomorphic to $S^{n-1}$, $n=\operatorname{dim} X$ and $d \theta$ a normalized standard measure on $\partial X$.

Denote by $\mathcal{P}(\partial X)$ a space of probability measures $\mu$ on $\partial X$ which is absolutely continuous with respect to $d \theta(\mu \ll d \theta)$ whose density function $p=p(\theta)$ is of $C^{0}$ and positive;

$$
\mathcal{P}(\partial X)=\left\{\mu=p(\theta) d \theta ; \int_{\partial X} p(\theta) d \theta=1, p \in C^{0}(\partial X), p(\theta)>0(\forall \theta \in \partial X)\right\} .
$$

Definition 5. Let $\mu \in \mathcal{P}(\partial X)$. Then, a function $\mathbf{B}_{\mu}: X \rightarrow \mathbf{R}$, called $\mu$-average Busemann function, is defined by

$$
\mathbf{B}_{\mu}(x)=\int_{\theta \in \partial X} B_{\theta}(x) d \mu(\theta) .
$$

Average Busemann function for any $\mu \in \mathcal{P}(\partial X)$ fulfills the following;

(i) For any $\mu \in \mathcal{P}(\partial X)$ each $\mathbf{B}_{\mu}$ is convex on $X$ and $\mathbf{B}_{\mu}\left(x_{o}\right)=0$. 
(ii) $\mathbf{B}_{\mu}(\gamma(t)) \rightarrow+\infty$, as $t \rightarrow \infty$, where $\gamma: \mathbf{R} \rightarrow X$ is an arbitrary geodesic in $X$ (Theorem 12).

(iii) $\mathbf{B}_{\mu}$ is Lipschitz continuous, in fact, $\left|\mathbf{B}_{\mu}(x)-\mathbf{B}_{\mu}(y)\right| \leq|d(x, y)|$ for $x, y \in X$.

(iv) The gradient vector field $\nabla \mathbf{B}_{\mu}$ is defined on $X$ as

$$
\left(\nabla \mathbf{B}_{\mu}\right)_{x}=\int_{\partial X}\left(\nabla B_{\theta}\right)_{x} d \mu(\theta), \quad x \in X
$$

and satisfies $\left|\left(\nabla \mathbf{B}_{\mu}\right)_{x}\right| \leq 1, x \in X$.

(v) the Hessian $\nabla d \mathbf{B}_{\mu}$ can be defined as $\mu$-average Hessian;

$$
\left(\nabla d \mathbf{B}_{\mu}\right)_{x}(u, v)=\int_{\partial X}\left(\nabla d B_{\theta}\right)_{x}(u, v) d \mu(\theta), \quad u, v \in T_{x} X x \in X,
$$

provided $(X, g)$ is of bounded Ricci curvature and moreover $\Delta B_{\theta}$, the Laplacian of $B_{\theta}$ together with $d\left(\Delta B_{\theta}\right)$ are uniformly bounded with respect to $x \in X$ and $\theta \in \partial X$. Here $\Delta f=-$ trace $\nabla d f$ for a $C^{2}$-function $f$ on $X$. This is derived from Bochner formula (see [23]). If $(X, g)$ is asymptotically harmonic ([34]), i.e., $\Delta B_{\theta} \equiv c$ for any $\theta$, and of bounded Ricci curvature, the average Hessian $\nabla d \mathbf{B}_{\mu}, \mu \in \mathcal{P}(\partial X)$ is defined.

Definition 6. Let $\mu \in \mathcal{P}(\partial X)$. A critical point of $\mu$-average Busemann function $\mathbf{B}_{\mu}$ is called a barycenter of $\mu$.

For a $C^{1}$-function $f: X \rightarrow \mathbf{R}, y \in X$ is called a critical point of $f$, if one of the following equivalent conditions holds;

(i) the differential of $f$ at $y$ vanishes along all directional vector, i.e.,

$$
\left.\frac{d}{d t}\right|_{t=0} f(x(t))=0
$$

for any $C^{1}$-curve $x(t)$ of $x(0)=y$,

(ii) the one-form $d f$, or the gradient vector field $\nabla f$ vanishes at $y$.

Observation. For $\mu=p(\theta) d \theta \in \mathcal{P}(\partial X), x \in X$ is a barycenter of $\mu$ if and only if $\left(d \mathbf{B}_{\mu}\right)_{x}(u)=0$ for any $u \in T_{x} X$, which is equivalent to stating that a measure $\tau$ defined by $\tau=\left(d B_{\theta}\right)_{x}(u) d \mu=$ $\left\langle\left(\nabla B_{\theta}\right)_{x}, u\right\rangle p(\theta) d \theta$ is a tangent vector to $\mathcal{P}(\partial X)$ at $\mu$ for each $u \in T_{x} X$.

Theorem 12. If, as is assumed, an Hadamard manifold $(X, g)$ satisfies visibility axiom and Busemann function is continuous with respect to any $\theta \in \partial X$. Then, every $\mu \in \mathcal{P}(\partial X)$ admits a barycenter.

Proof. This theorem is proved by Besson, Courtois and Gallot in [8] by showing $\mathbf{B}_{\mu}(\gamma(t)) \rightarrow \infty$ as $t \rightarrow+\infty$ along any geodesic $\gamma$ of $X$. However, they assume that all probability measures on $\partial X$ are without atom and an Hadamard manifold $(X, g)$ is of special type, i.e., a rank one symmetric space of non-compact type. We restrict the space of probability measures as $\mathcal{P}(\partial X)$. However, we relax 
the assumptions concerning an Hadamard manifold $(X, g)$ and then, assume only that $(X, g)$ satisfies visibility axiom and Busemann function is continuous with respect to any $\theta \in \partial X$.

Let $C>0$ be a constant and set $A_{C}=\left\{y \in X ; \mathbf{B}_{\mu}(y) \leq C\right\}$. $A_{C}$ is a convex set and $x_{o} \in A_{C}$, since $\mathbf{B}_{\mu}$ is convex and $\mathbf{B}_{\mu}\left(x_{o}\right)=0$. Note $A_{C}$ contains a geodesic ball $\left\{y \in X ; d\left(y, x_{o}\right) \leq C / 2\right\}$ since $\mathbf{B}_{\mu}$ is Lipschitz. Let $\mu \in \mathcal{P}(\partial X)$ and $\gamma$ be a geodesic satisfying $\gamma(0)=x_{o}$ and $[\gamma]=\theta$. Then it is possible to verify $\lim _{t \rightarrow+\infty} \mathbf{B}_{\mu}(\gamma(t))=+\infty$ in the following steps;

Step I. Since Busemann function $B_{\theta}$ is convex and $B_{\theta}\left(x_{0}\right)=0$ for any $\theta$, we have

$$
d\left(\gamma\left(t_{1}\right), x_{0}\right) B_{\theta}\left(\gamma(t) \geq d\left(\gamma(t), x_{0}\right) B_{\theta}\left(\gamma\left(t_{1}\right)\right.\right.
$$

in other words

$$
t_{1} B_{\theta}(\gamma(t)) \geq t B_{\theta}\left(\gamma\left(t_{1}\right)\right) \quad\left(0 \leq t_{1} \leq t\right) .
$$

In fact, if we set $a=t_{1} / t$, then, $0 \leq a \leq 1$ and we have $t_{1}=(1-a) 0+a t$. The Convex function $B_{\theta}(\gamma(t))$ fulfills

$$
B_{\theta}\left(\gamma\left(t_{1}\right)\right) \leq(1-a) B_{\theta}(\gamma(0))+a B_{\theta}(\gamma(t))=a B_{\theta}(\gamma(t))
$$

that is

$$
B_{\theta}\left(\gamma\left(t_{1}\right)\right) \leq a B_{\theta}(\gamma(t))
$$

which is just (31).

Step II. Fix $t_{1}>0$ of Step I. Take an arbitrary $\theta_{0} \in \partial X$ and fix it. Let $\gamma$ be a geodesic of $\gamma(0)=x_{0}$ and $[\gamma]=\theta_{0}$. For any $t>0$ set

$$
J_{\theta_{0}}(t):=\left\{\theta \in \partial X ; B_{\theta}(\gamma(t)) \leq 0\right\} .
$$

We show that there exists a $t_{1} \in(0, \infty)$ such that $\mu\left(J_{\theta_{0}}\left(t_{1}\right)\right)<1$, as follows.

Since $B_{\theta}(x)$ is continuous with respect to $\theta$, the set $J_{\theta_{0}}(t)$ is compact in $\partial X$. We see $\theta_{0} \in J_{\theta_{0}}(t)$. From (32) it holds

$$
J_{\theta_{0}}(t) \subset J_{\theta_{0}}\left(t_{1}\right) \quad\left(0 \leq t_{1} \leq t\right) .
$$

It follows then

$$
\bigcap_{t \in[0, \infty)} J_{\theta_{0}}(t)=\left\{\theta_{0}\right\}
$$

because from the visibility axiom, we have from Proposition 3.4 for any $\theta \in \partial X$ such that $\theta \neq \theta_{0}$ $\lim _{t \rightarrow \infty} B_{\theta}(\gamma(t))=+\infty$. Moreover, from (33) for the $\mu$ we find

$$
\lim _{t \rightarrow \infty} \mu\left(J_{\theta_{0}}(t)\right)=\mu\left(\bigcap_{t \in[0, \infty)} J_{\theta_{0}}(t)\right)=\mu\left(\left\{\theta_{0}\right\}\right)=0 .
$$

Therefore, we have a $t_{1} \in(0, \infty)$ such that $\mu\left(J_{\theta_{0}}\left(t_{1}\right)\right)<1$. Here, notice $\mu\left(J_{\theta_{0}}(t)\right) \leq \mu\left(J_{\theta_{0}}\left(t_{1}\right)\right)<1$ for any $t \geq t_{1}>0$. 
Step III. Let $K$ be a compact subset of $\partial X \backslash J_{\theta_{0}}\left(t_{1}\right)$ satisfying $\mu(K)>0$. It is possible to choose such a $K$. Then, from (33) it holds for any $t \geq t_{1}$ that $K \subset \partial X \backslash J_{\theta_{0}}(t)$, and $B_{\theta}(\gamma(t)) \geq 0$ for any $\theta \in \partial X \backslash J_{\theta_{0}}(t)$. So,

$$
\begin{aligned}
\int_{\partial X} B_{\theta}(\gamma(t)) d \mu(\theta) & =\int_{J_{\theta_{0}}(t)} B_{\theta}(\gamma(t)) d \mu(\theta)+\int_{\partial X \backslash J_{\theta_{0}}(t)} B_{\theta}(\gamma(t)) d \mu(\theta) \\
& \geq \int_{J_{\theta_{0}}(t)} B_{\theta}(\gamma(t)) d \mu(\theta)+\int_{K} B_{\theta}(\gamma(t)) d \mu(\theta)
\end{aligned}
$$

Since $K$ is compact, we choose a constant $C>0$ satisfying

$$
B_{\theta}\left(\gamma\left(t_{1}\right)\right) \geq C>0, \quad \forall \theta \in K
$$

From (32), we have

$$
\int_{\partial X} B_{\theta}(\gamma(t)) d \mu(\theta) \geq \frac{t}{t_{1}} \int_{J_{\theta_{0}}(t)} B_{\theta}\left(\gamma\left(t_{1}\right)\right) d \mu(\theta)+C \frac{t}{t_{1}} \mu(K) .
$$

To estimate the first term of the RHS we choose $D \geq 0$ satisfying

$$
B_{\theta}\left(\gamma\left(t_{1}\right)\right) \geq-\sup \left\{\left|B_{\theta}\left(\gamma\left(t_{1}\right)\right)\right| ; \theta \in \partial X\right\}=-D
$$

In fact, since $\partial X$ is compact, $B_{\theta}\left(\gamma\left(t_{1}\right)\right)$, as a continuous function of $\theta$, is bounded with respect to $\theta$. Therefore, the above is written as

$$
\int_{\partial X} B_{\theta}(\gamma(t)) d \mu(\theta) \geq \frac{t}{t_{1}}\left(-D \mu\left(J_{\theta_{0}}(t)\right)+C \mu(K)\right) .
$$

We let $t \rightarrow+\infty$ and then, from (34) we have

$$
\lim _{t \rightarrow \infty} \mathbf{B}_{\mu}(\gamma(t))=\lim \int_{\partial X} B_{\theta}(\gamma(t)) d \mu(\theta)=+\infty
$$

from which it follows that the closed set $A_{C}$ is bounded and hence is compact. Therefore, $\mathbf{B}_{\mu}$ admits a minimal point $x \in X$, namely, $x$ is a barycenter of $\mu$.

Proposition 6. Let $(X, g)$ be an Hadamard manifold of bounded Ricci curvature. If $(X, g)$ is asymptotically harmonic, then the following holds; If there exists $\mu_{0} \in \mathcal{P}(\partial X)$ such that $\mu$-average Hessian $\nabla d \mathbf{B}_{\mu_{0}}$ is positive definite at every point in $X$, then, for any $\mu \in \mathcal{P}(\partial X) \mu$-average Hessian $\nabla d \mathbf{B}_{\mu}$ is also positive definite at every point in $X$.

Proof. Let $x \in X$ and $u \in T_{x} X$. Then, for a geodesic $\gamma$ in $X, \gamma(0)=x, \dot{\gamma}(0)=u$ we have

$$
\begin{aligned}
\left(\nabla d \mathbf{B}_{\mu_{0}}\right)_{x}(u, u) & =\left.\frac{d^{2}}{d t^{2}}\right|_{t=0} \mathbf{B}_{\mu_{0}}(\gamma(t))=\left.\int_{\theta \in \partial X} \frac{d^{2}}{d t^{2}}\right|_{t=0} B_{\theta}(\gamma(t)) d \mu_{0}(\theta) \\
& =\int_{\partial X}\left(\nabla d B_{\theta}\right)_{x}(u, u) d \mu_{0}(\theta) .
\end{aligned}
$$

Similarly for any $\mu \in \mathcal{P}(\partial X)$, we have

$$
\begin{aligned}
\left(\nabla d \mathbf{B}_{\mu}\right)_{x}(u, u) & =\left.\frac{d^{2}}{d t^{2}}\right|_{t=0} \mathbf{B}_{\mu}(\gamma(t))=\left.\int_{\theta \in \partial X} \frac{\partial^{2}}{\partial t^{2}}\right|_{t=0} B_{\theta}(\gamma(t)) d \mu(\theta) \\
& =\int_{\partial X}\left(\nabla d B_{\theta}\right)_{x}(u, u) d \mu(\theta) .
\end{aligned}
$$


Let $C=\min _{\theta \in \partial X} \frac{d \mu}{d \mu_{0}}(\theta)>0$. It is concluded then from the above

$$
\left(\nabla d \mathbf{B}_{\mu}\right)_{x}(u, u) \geq C\left(\nabla d \mathbf{B}_{\mu_{0}}\right)_{x}(u, u)>0, \quad \forall u \in T_{x} X(\neq 0), x \in X .
$$

Theorem 13. Let $(X, g)$ be an Hadamard manifold satisfying the above assumptions. Then, any $\mu \in$ $\mathcal{P}(\partial X)$ admits a unique barycenter.

In fact, Theorem 12 asserts an existence of a barycenter for any $\mu$. From Proposition 6 a barycenter must be unique, since, if, otherwise, $\mu$ admits barycenters $y_{1}, y_{2}, y_{1} \neq y_{2}$, then $f(t):=\mathbf{B}_{\mu}(\gamma(t))$ along a geodesic $\gamma: \mathbf{R} \rightarrow X$ joining $y_{1}$ and $y_{2}$ satisfies $f^{\prime}(0)=f^{\prime}(d)=0\left(d=d\left(y_{1}, y_{2}\right)\right)$ and $f^{\prime \prime}(t)>0$, $t \in[0, d]$ because of the positive definiteness of $\mu$-average Hessian (35). So, $f(t)$ must be constant along $\gamma$. This contradicts property (ii) of $\mu$-average Busemann function. Hence uniqueness is proved.

Proposition 7 (average Busemann cocycle formula). Let $\phi$ be an isometry of an Hadamard manifold $(X, g)$. Then for any $\mu \in \mathcal{P}(\partial X)$

$$
\mathbf{B}_{\mu}\left(\phi^{-1} x\right)=\mathbf{B}_{(\hat{\phi})_{\sharp \mu}}(x)+\mathbf{B}_{\mu}\left(\phi^{-1} x_{o}\right) .
$$

Proof. Integrate the Busemann cocycle formula (28)

$$
B_{\theta}\left(\phi^{-1}(x)\right)=B_{\hat{\phi}(\theta)}(x)+B_{\theta}\left(\phi^{-1} x_{o}\right)
$$

for the inverse isometry $\phi^{-1}$ with respect to a measure $\mu$. We then get (36).

From Theorem 13 we define a map, called barycenter map

$$
\text { bar : } \mathcal{P}(\partial X) \rightarrow X ; \quad \mu \mapsto y,
$$

by assigning a barycenter $y$ to $\mu$.

Example 5. The standard measure $d \theta$ has $\operatorname{bar}(d \theta)=x_{o}$, the base point as its barycenter. In fact, we observe

$$
\int_{\partial X}\left\langle\left(\nabla B_{\theta}\right)_{x_{o}}, u\right\rangle d \theta=0, \quad \forall u \in T_{x_{o}} X,
$$

since $d \theta=\left(\beta_{x_{o}}\right)_{\sharp}(d \theta)_{x_{o}}$ is the push-forward of the spherical measure $(d \theta)_{x_{o}}$ of $S_{x_{o}} X$, where $\beta_{x_{o}}$ is a map given in (27), and one has $\beta_{x_{o}}\left(-\left(\nabla B_{\theta}\right)_{x_{o}}\right)=\theta$ so that $\beta_{x_{o}}(v)=\theta$ implies $v=-\left(\nabla B_{\theta}\right)_{x_{o}}$. Then the LHS of the above is written as

$$
\int_{v \in S_{x_{o}} X}\left\langle\left(\nabla B_{\beta_{x_{o}}(v)}\right)_{x_{o}}, u\right\rangle d \theta_{x_{o}}(v)=-\int_{v \in S_{x_{o}} X}\langle v, u\rangle d \theta_{x_{o}}(v)=0 .
$$

Here, the last integration is derived from a standard formula on $S^{n-1}$ which is described as $\sum_{i=1}^{n}\left(\theta^{i}\right)^{2}=$ 1 with respect to the standard coordinates $\theta=\left(\theta^{1}, \ldots, \theta^{n}\right) \in \mathbf{R}^{n}$;

$$
\int_{\theta \in S^{n-1}} \theta^{i}(d \theta)_{S^{n-1}}=0, \quad i=1, \cdots, n .
$$




\subsection{Barycenter Map}

In this subsection we will verify that the barycenter map (37) enjoys a fibration over an Hadamard manifold $X$ in terms of Fisher metric $G$. Before giving a detailed argument, we prepare some special probability measures on $\partial X$ which play a crucial role in the barycenter map, that is, Poisson kernel measures. Here, Poisson kernel is a fundamental solution of Dirichlet problem at $\partial X$; given a $C^{0}$-function $f$ on $\partial X$, find a function $u$ on $X$ which satisfies the Laplace equation $\Delta u=0$ and the boundary condition $\lim _{x \rightarrow \theta} u(x)=f(\theta)$ for $\theta \in \partial X$.

Definition 7 ([3,35]). A function $P_{\theta}(x)=P(x, \theta)$ on $X$ is called a Poisson kernel, normalized at $x_{o}$, for $\theta \in \partial X$ if it satisfies

(i) $\Delta P(x, \theta)=0$ and $P(x, \theta)>0$ for any $x \in X$ and $\theta \in \partial X$.

(ii) $P\left(x_{o}, \theta\right)=1$ for any $\theta \in \partial X$.

(iii) for any $\theta \in \partial X, P(x, \theta) \in C^{0}(X \cup \partial X \backslash\{\theta\})$ as an extension function on $X \cup \partial X$ and $\lim _{x \rightarrow \theta_{1}} P(x, \theta)=0$ for $\theta_{1} \neq \theta$.

The solution $u=u(x)$ of the Dirichlet problem on $\partial X$ is described as an integration form;

$$
u(x)=\int_{\theta \in \partial X} P(x, \theta) f(\theta) d \theta
$$

so, $P(x, \theta) d \theta \in \mathcal{P}(\partial X)$ for each $x \in X$.

Example 6. On a real hyperbolic space $H^{n}(\mathbf{R})$ of standard hyperbolic metric of Poicaré ball model, the Poisson kernel is given by

$$
P(x, \theta)=\left(\frac{1-|x|^{2}}{|x-\theta|^{2}}\right)^{n-1}, \quad \theta \in \partial X=S^{n-1} .
$$

Example 7. The Poisson integral formula, well known in potential theory, is for a bounded harmonic function $h=h(z), z=r e^{i \varphi} \in\{z \in \mathbf{C}|| z \mid \leq 1\}$

$$
h\left(r e^{i \varphi}\right)=\frac{1}{2 \pi} \int_{0 \leq \theta \leq 2 \pi} \frac{1-r^{2}}{1-2 r \cos (\varphi-\theta)+r^{2}} f\left(e^{i \theta}\right) d \theta,
$$

where $f$ is a bounded function on $S^{1}$. The kernel function $\left(1-r^{2}\right) /\left(1-2 r \cos (\varphi-\theta)+r^{2}\right)$ is just the Poisson kernel $P(z, \theta)=\left(1-|z|^{2}\right) /|z-\theta|^{2}$ of the hyperbolic plane $H^{2}(\mathbf{R})$. See, for example [20].

Definition 8. A Poisson kernel on an Hadamard manifold $(X, g)$ is called Busemann-Poisson kernel, when it has the following form

$$
P(x, \theta)=\exp \left\{-Q B_{\theta}(x)\right\}, \quad x \in X, \theta \in \partial X,
$$

where $Q>0$ is volume entropy of $(X, g)$, the exponential growth rate of the volume of $(X, g)$

$$
Q=\lim _{r \rightarrow \infty} \frac{1}{r} \log \operatorname{vol} B(x, r)
$$

for a geodesic ball $B(x, r)$. 
Remark 5. For volume entropy refer to [8] in which the following theorem, Theorem of Manning, ([7]) is cited; if $Q_{\text {top }}$ denotes the topological entropy of a compact Riemannian manifold $Y$ of non-positive curvature, then one has

(i) $Q(\tilde{Y}) \leq Q_{\text {top }}(Y)$,

(ii) $Q(\tilde{Y})=Q_{\text {top }}(Y)$, provided the curvature of $Y$ is negative or zero.

Here, $\tilde{Y}$ is the universal covering space of $Y$ and the topological entropy $Q_{\text {top }}(Y)$ is defined by

$$
Q_{\text {top }}(Y)=\lim _{R \rightarrow \infty} \frac{1}{R} \log (\sharp\{\gamma \mid \ell(\gamma) \leq R\}),
$$

where $\gamma$ denotes a periodic geodesic in $Y$ of length $\ell(\gamma)$ and $\sharp\{\gamma \mid \ell(\gamma) \leq R\}$ denotes the number of periodic geodesics of length not greater than $R$.

For example $Q=0$ for a Euclidean space and $Q=n-1$ for a real hyperbolic space $H^{n}(\mathbf{R})$ of standard hyperbolic metric.

Remark 6. Any Damek-Ricci space admits a Busemann-Poisson kernel (refer to [4]). See also [8] for a rank one symmetric space of non-compact type which is just a member of Damek-Ricci spaces, as observed by using Iwasawa decomposition of isometry groups.

Theorem 14. Let $(X, g)$ be an Hadamard manifold satisfying the assumptions in Theorem 12 and Proposition 6. If $(X, g)$ admits a Busemann-Poisson kernel, then, for $\mu_{x}:=P(x, \theta) d \theta \in \mathcal{P}(\partial X)$

(i) $\operatorname{bar}\left(\mu_{x}\right)=x$ for any $x \in X$ and

(ii) at any point $y \in X,\left(\nabla d \mathbf{B}_{\mu_{x}}\right)_{y}$ is positive definite.

The statement (i) is shown in [8]. From (i) the barycenter map bar is onto.

Definition 9. Let $\mu=p(\theta) d \theta \in \mathcal{P}(\partial X)$ and $x \in X$ be a barycenter of $\mu$. We define a linear map

$$
\nu_{x}^{\mu}: T_{x} X \rightarrow T_{\mu} \mathcal{P}(\partial X) ; \quad u \mapsto \nu_{x}^{\mu}(u)=\left(d B_{\theta}\right)_{x}(u) \mu=\left\langle\left(\nabla B_{\theta}\right)_{x}, u\right\rangle p(\theta) d \theta .
$$

Notice that the map $\nu_{x}^{\mu}$ is injective.

Proof of Theorem 14. (i) Let $u \in T_{x} X$ and $x(t)$ a $C^{1}$-curve in $X$ such that $x(0)=x, \dot{x}(0)=u$. Differentiate $\int_{\partial X} P(x(t), \theta) d \theta \equiv 1$ as

$$
\begin{aligned}
0 & =\left.\frac{d}{d t}\right|_{t=0} \int P(x(t), \theta) d \theta \\
& =\left.\int \frac{\partial}{\partial t}\right|_{t=0} \exp \left\{-Q B_{\theta}(x(t))\right\} d \theta \\
& =\int-Q\left(d B_{\theta}\right)_{x}(\dot{x}(0)) \exp \left\{-Q B_{\theta}(x(0))\right\} d \theta \\
& =-\left.Q \frac{d}{d t}\right|_{t=0} \int_{\partial X} B_{\theta}(x(t)) d \mu_{x}=-Q\left(d \mathbf{B}_{\mu_{x}}\right)_{x}(u) .
\end{aligned}
$$

So, $x=$ bar $\mu_{x}$. 
For a proof of (ii) we first show

Assertion 4. The measure $\mu_{x}$ satisfies

$$
\left(\nabla d \mathbf{B}_{\mu_{x}}\right)_{x}(u, v)=Q G_{\mu_{x}}\left(\nu_{x}^{\mu_{x}}(u), \nu_{x}^{\mu_{x}}(v)\right), \quad u, v \in T_{x} X
$$

in terms of the Fisher metric $G$, where $\nu_{x}^{\mu_{x}}$ is the linear map defined in (38).

It suffices to show this in case of $u=v$. Let $\gamma$ be a geodesic in $X$ satisfying $\gamma(0)=x, \dot{\gamma}(0)=u$. Then we have

$$
0=\left.\frac{d^{2}}{d t^{2}}\right|_{t=0} \int_{\partial X} P(\gamma(t), \theta) d \theta
$$

However, this is

$$
\begin{aligned}
& \left.\int_{\partial X} \frac{\partial^{2}}{\partial t^{2}}\right|_{t=0} \exp \left\{-Q B_{\theta}(\gamma(t))\right\} d \theta \\
= & -Q \int_{\partial X}\left\{\left(\nabla d B_{\theta}\right)_{x}(u, u)-Q\left\{\left(d B_{\theta}\right)_{x}(u)\right\}^{2}\right\} \exp \left\{-Q B_{\theta}(x)\right\} d \theta \\
= & -Q\left\{\left(\nabla d \mathbf{B}_{\mu_{x}}\right)_{x}(u, u)-Q G_{\mu_{x}}\left(\nu_{x}^{\mu_{x}}(u), \nu_{x}^{\mu_{x}}(u)\right)\right\}
\end{aligned}
$$

showing (39).

From this assertion (ii) is proved as follows. At $y \in X$ we have, since $\left(\nabla d B_{\theta}\right)_{y}(\cdot, \cdot)$ is positive semi-definite,

$$
\begin{aligned}
\left(\nabla d \mathbf{B}_{\mu_{x}}\right)_{y}(u, u) & =\int\left(\nabla d B_{\theta}\right)_{y}(u, u) d \mu_{x}(\theta)=\int\left(\nabla d B_{\theta}\right)_{y}(u, u) P(x, \theta) d \theta \\
& \geq C \int\left(\nabla d B_{\theta}\right)_{y}(u, u) P(y, \theta) d \theta=C\left(\nabla d \mathbf{B}_{\mu_{y}}\right)_{y}(u, u)
\end{aligned}
$$

for any $u \in T_{y} X$, where $C=\inf _{\theta \in \partial X} P(x, \theta) / P(y, \theta)>0$.

Now we will investigate the map bar $: \mathcal{P}(\partial X) \rightarrow X$.

Theorem 15. The barycenter map bar $: \mathcal{P}(\partial X) \rightarrow X$ gives a projection of a fibre space whose total space is $\mathcal{P}(\partial X)$ and base space is $X$ with fibres $\operatorname{bar}^{-1}(x)$ over $x \in X$. In fact, let $x \in X$ and $\mu \in$ $\operatorname{bar}^{-1}(x)$. Then

$$
T_{\mu} \mathcal{P}(\partial X)=T_{\mu} \operatorname{bar}^{-1}(x) \oplus \operatorname{Im} \nu_{x}^{\mu} \quad\left(\operatorname{dim} \operatorname{Im} \nu_{x}^{\mu}=n\right),
$$

as an orthogonal direct sum of the vertical subspace $T_{\mu} \operatorname{bar}^{-1}(x)$ and the horizontal subspace $\operatorname{Im} \nu_{x}^{\mu}$ with respect to Fisher metric $G_{\mu}$.

This orthogonal decomposition indicates that $N=\left\{N_{\mu}=\operatorname{Im} \nu_{x}^{\mu} ; \mu \in \operatorname{bar}^{-1}(x)\right\}$ distributes a normal bundle to each fibre $\operatorname{bar}^{-1}(x), x \in X$. Notice that $\operatorname{bar}^{-1}(x)$ is path-connected, since, for $\mu$, $\mu_{1} \in \operatorname{bar}^{-1}(x)(1-t) \mu+t \mu_{1}, 0 \leq t \leq 1$, also belongs to $\operatorname{bar}^{-1}(x)$. 
We will show that the vertical subspace $T_{\mu} \operatorname{bar}^{-1}(x)$ is orthogonal to $N_{\mu}=\operatorname{Im} \nu_{x}^{\mu}$. Let $u \in T_{x} X$ and $\tau \in T_{\mu} \operatorname{bar}^{-1}(x)$ and take $\mu(t)=\mu+t \tau$ for sufficiently small $|t|$. Then, $\mu(t) \in \operatorname{bar}^{-1}(x)$. So, for a sufficiently small $|t|$, we have

$$
\begin{aligned}
0 & =\int\left(d B_{\theta}\right)_{x}(u) d \mu(t)(\theta)=\int\left(d B_{\theta}\right)_{x}(u) d(\mu+t \tau)(\theta) \\
& =\int\left(d B_{\theta}\right)_{x}(u) d \mu(\theta)+t \int\left(d B_{\theta}\right)_{x}(u) d \tau(\theta) \\
& =t \int\left(d B_{\theta}\right)_{x}(u) d \tau(\theta)=t G_{\mu}\left(\nu_{x}^{\mu}(u), \tau\right) .
\end{aligned}
$$

This means the orthogonality of $T_{\mu} \operatorname{bar}^{-1}(x)$ and $N_{\mu}=\operatorname{Im} \nu_{x}^{\mu}$.

Since the image $N_{\mu}=\operatorname{Im} \nu_{x}^{\mu}$ is a finite dimensional subspace of $T_{\mu} \mathcal{P}(\partial X)$, the direct sum decomposition is easily shown and so we skip.

\subsection{Fibres $\operatorname{bar}^{-1}(x)$ and Geodesics}

We discussed in Section 3 several properties and propositions of geodesics on a space of probability measures. In this section we will investigate under which condition a geodesic of $\mathcal{P}(\partial X)$ is contained in a fibre $\operatorname{bar}^{-1}(x)$.

Theorem 16. Let $(X, g)$ be an Hadamard manifold satisfying the assumptions in Theorem 12 and Proposition 6, and admitting a Busemann-Poisson kernel.

Let $\mu \in \operatorname{bar}^{-1}(x)$ and $\tau \in T_{\mu} \operatorname{bar}^{-1}(x),|\tau|_{G, \mu}=1$. Then a geodesic $\mu(t)=\exp _{\mu} t \tau$ entirely belongs to $\operatorname{bar}^{-1}(x)$ for any $t$ at which $\mu(t)$ is well-defined, if and only if $\tau$ fulfills $H_{\mu}(\tau, \tau)=0$.

Here $H$ is the second fundamental form of a submanifold $\operatorname{bar}^{-1}(x)$ of the ambient space $\mathcal{P}(\partial X)$ (see Equation (5) in Section 2).

Proof. From Theorem 10, Section 3 the geodesic $\mu(t)$ is given by

$$
\mu(t)=\left(\cos \frac{t}{2}+\sin \frac{t}{2} \frac{d \tau}{d \mu}(\theta)\right)^{2} \mu .
$$

Then $\mu(t) \in \operatorname{bar}^{-1}(x)$ for all $t$ if and only if for any $u \in T_{x} X$

$$
0=\int_{\theta \in \partial X}\left(d B_{\theta}\right)_{x}(u) d \mu(t)(\theta)
$$

However the RHS is

$$
\cos ^{2} \frac{t}{2} \int\left(d B_{\theta}\right)_{x}(u) d \mu(\theta)+2 \cos \frac{t}{2} \sin \frac{t}{2} \int\left(d B_{\theta}\right)_{x}(u) \frac{d \tau}{d \mu}(\theta) d \mu(\theta)+\sin ^{2} \frac{t}{2} \int\left(d B_{\theta}\right)_{x}(u)\left(\frac{d \tau}{d \mu}\right)^{2}(\theta) d \mu(\theta)
$$

for all $t$. Since $\mu \in \operatorname{bar}^{-1}(x)$ and $\tau \in T_{\mu} \operatorname{bar}^{-1}(x)$, this is equivalent to

$$
0=\int\left(d B_{\theta}\right)_{x}(u)\left(\frac{d \tau}{d \mu}\right)^{2}(\theta) d \mu(\theta)
$$


which is reduced by the aid of Levi-Civita connection formula to

$$
0=\int\left(d B_{\theta}\right)_{x}(u)\left(\frac{d \nabla_{\tau} \tau}{d \mu}\right)(\theta) d \mu(\theta)=-2 G_{\mu}\left(\nu_{x}^{\mu}(u), \nabla_{\tau} \tau\right)
$$

which means that $H(\tau, \tau)=0$. Conversely, if $\tau$ satisfies $H(\tau, \tau)=0$, then it is easy to see that $\mu(t)=\exp _{\mu} t \tau$ belongs to the fibre $\operatorname{bar}^{-1}(x)$ by following reversely the above argument.

Theorem 17. Let $\mu, \mu^{*} \in \operatorname{bar}^{-1}(x), x \in X\left(\mu \neq \mu^{*}\right)$. Then, a geodesic $\mu(t)$ joining $\mu$ and $\mu^{*}$ lies entirely on $\operatorname{bar}^{-1}(x)$ if and only if

$$
\int_{\partial X}\left(d B_{\theta}\right)_{x}(u) \sqrt{\frac{d \mu^{*}}{d \mu}}(\theta) d \mu(\theta)=0, \quad \forall u \in T_{x} X .
$$

Proof. The geodesic $\mu(t)$ joining $\mu$ and $\mu^{*}$ is written from Theorem 11 by $\exp _{\mu} t \tau$ of an initial vector

$$
\tau=\frac{1}{\sin \frac{\ell}{2}}\left\{\sqrt{\frac{d \mu^{*}}{d \mu}}(\theta)-\cos \frac{\ell}{2}\right\} \mu, \quad \ell=\ell(\mu, \mu *)>0 .
$$

Then, $\mu(t)$ lies on $\operatorname{bar}^{-1}(x)$ if and only if the following conditions hold, that is, $\tau$ is tangent to $\operatorname{bar}^{-1}(x)$, namely,

$$
G_{\mu}\left(\nu_{x}^{\mu}(u), \tau\right)=0
$$

for any $u \in T_{x} X$, and that

$$
H(\tau, \tau)=-\frac{1}{2} \int_{\partial X}\left(d B_{\theta}\right)_{x}(u)\left(\frac{d \tau}{d \mu}\right)^{2}(\theta) d \mu(\theta)=0 .
$$

Equation (42) is equivalent to (40), since $\tau$ is given by (41). On the other hand, (43) is written as

$$
\begin{aligned}
0 & =\int_{\partial X}\left(d B_{\theta}\right)_{x}(u)\left(\sqrt{\frac{d \mu^{*}}{d \mu}}(\theta)-\cos \frac{\ell}{2}\right)^{2} d \mu(\theta) \\
& =-2 \cos \frac{\ell}{2} \int_{\partial X}\left(d B_{\theta}\right)_{x}(u) \sqrt{\frac{d \mu^{*}}{d \mu}}(\theta) d \mu(\theta)
\end{aligned}
$$

for any $u \in T_{x} X$. This condition is also (40), so we get Theorem 17.

Example 8. Let $\mu=d \theta$. Then, $\operatorname{bar}(d \theta)=x_{o}$, as seen in Example 5. We exhibit tangent vectors $\tau, \tau_{1}$ at $d \theta$ satisfying $H(\tau, \tau)=0$, whereas $H\left(\tau_{1}, \tau_{1}\right) \neq 0$, as follows;

(i) Identify $\partial X$ with $S_{x_{o}} X \cong S^{n-1}$ via $\beta_{x_{o}}$, and $d \theta$ with $(d \theta)_{x_{o}}$. Choose on $S^{n-1}$ a function $q=$ $q(\theta)=\theta^{i} \theta^{j}, i \neq j$ and define $\tau=q(\theta) d \theta$ as a measure on $\partial X$. Then, $\tau \in T_{d \theta} \mathcal{P}(\partial X)$. Moreover, $\tau \in T_{d \theta} \operatorname{bar}^{-1}\left(x_{o}\right)$, since $G_{d \theta}\left(\nu_{x_{o}}^{d \theta}(u), \tau\right)=0$ for any $u \in T_{x_{o}} X$ and $H(\tau, \tau)=0$. These are directly from the integral formulae; $\int_{S^{n-1}} \theta^{i} \theta^{j} \theta^{k}(d \theta)_{x_{o}}=0, \int_{S^{n-1}}\left(\theta^{i} \theta^{j}\right)^{2} \theta^{k}(d \theta)_{x_{o}}=0$ for any $k=1, \ldots, n$. By normalizing $\tau^{\prime}=\tau /|\tau|_{G}$ in terms of $G$, from Theorem $16 \gamma(t)=\exp _{d \theta} t \tau^{\prime}$ gives a geodesic lying on $\operatorname{bar}^{-1}\left(x_{o}\right)$.

(ii) Let $q_{1}=q_{1}(\theta)$ is a function on $S^{n-1}, n \geq 3$, defined by $q_{1}(\theta)=\theta^{1} \theta^{2} \theta^{3}+\theta^{2} \theta^{3}$ and set $\tau_{1}=q_{1}(\theta) d \theta$. Then $\left(\gamma_{1}\right)(t)=\exp _{d \theta} t \tau_{1}^{\prime}, \tau_{1}^{\prime}=\tau_{1} /\left|\tau_{1}\right|_{G}$, is a geodesic being not completely on the fibre $\operatorname{bar}^{-1}\left(x_{o}\right)$. 


\section{Barycentrically Associated Maps}

Let $\phi$ be an isometry of an Hadamard manifold $(X, g)$. Then, from the average Busemann cocycle formula (36).

Theorem 18 ([8]). For any isometry $\phi$ of $(X, g)$, we have

$$
\operatorname{bar}\left(\hat{\phi}_{\sharp} \mu\right)=\phi(\operatorname{bar}(\mu)), \quad \mu \in \mathcal{P}(\partial X) .
$$

Proof. Let $y=\operatorname{bar}\left(\hat{\phi}_{\sharp} \mu\right)$. Then $\left(d \mathbf{B}_{\hat{\phi}_{\sharp}}\right)_{y}(u)=0$ for any $u \in T_{y} X$, namely, due to (36) $\phi^{-1} y$ turns out to be a critical point of $\mathbf{B}_{\mu}$, that is, $y=\phi(\operatorname{bar}(\mu))$, so (44) is obtained.

Definition 10. Let $\Phi: \partial X \rightarrow \partial X$ be a homeomorphism of $\partial X$. Then, a bijective map $\phi: X \rightarrow X$ is said to be barycentrically associated to $\Phi$, if $\Phi$ and $\phi$ satisfy the relation bar $\circ \Phi=\phi \circ$ bar, that is, $\operatorname{bar}(\Phi(\mu))=\phi(\operatorname{bar}(\mu))$ for any $\mu \in \mathcal{P}(\partial X)$.

Now we are ready to give a proof of Theorem 6.

Proof of Theorem 6. From the statement of the theorem, diagram (6) asserts for any $x \in X$, i.e.,

$$
\Phi_{\sharp}\left(\nu_{x}^{\mu_{x}}(u)\right)=\nu_{\varphi x}^{\mu_{\varphi x}}\left(\left(\varphi_{*}\right)_{x}(u)\right), \quad \forall u \in T_{x} X,
$$

namely

$$
\Phi_{\sharp}\left(\left(d B_{\theta}\right)_{x}(u) \mu_{x}(\theta)\right)=\left(d B_{\theta}\right)_{\varphi x}\left(\left(\varphi_{*}\right)_{x} u\right) \mu_{\varphi x}(\theta)
$$

for $\mu_{x}=\Sigma(x)$, where $\Sigma: X \rightarrow \mathcal{P}(\partial X)$ is a cross section whose existence is assumed in the theorem. We write (46) as

$$
\left(d B_{\Phi^{-1} \theta}\right)_{x}(u) \Phi_{\sharp} \mu_{x}=\left(d B_{\theta}\right)_{\varphi x}\left(\left(\varphi_{*}\right)_{x} u\right) \mu_{\varphi x} .
$$

Since another diagram (6) implies $\Phi_{\sharp} \mu_{x}=\mu_{\varphi x}$, we have

$$
\left(d B_{\Phi^{-1} \theta}\right)_{x}(u) \mu_{\varphi x}=\left(d B_{\theta}\right)_{\varphi x}\left(\left(\varphi_{*}\right)_{x} u\right) \mu_{\varphi x}
$$

that is,

$$
\left(d B_{\Phi^{-1} \theta}\right)_{x}(u)=\left(d B_{\theta}\right)_{\varphi x}\left(\left(\varphi_{*}\right)_{x} u\right), \quad u \in T_{x} X, \forall \theta \in \partial X
$$

or

$$
\left\langle\left(\nabla B_{\Phi^{-1} \theta}\right)_{x}, u\right\rangle=\left\langle\left(\nabla B_{\theta}\right)_{\varphi x},\left(\varphi_{*}\right)_{x} u\right\rangle \quad u \in T_{x} X, \forall \theta \in \partial X
$$

Using the formal adjoint $\left(\left(\varphi_{*}\right)_{x}\right)^{*}$ of $\left(\varphi_{*}\right)_{x}$, we write the above as

$$
\left(\nabla B_{\Phi^{-1} \theta}\right)_{x}=\left(\left(\varphi_{*}\right)_{x}\right)^{*}\left(\nabla B_{\theta}\right)_{\varphi x} \quad u \in T_{x} X, \forall \theta \in \partial X
$$

Let $v \in S_{\varphi x} X$ be a unit tangent vector at $\varphi x$ and choose $\theta \in \partial X$ such that $\left(\nabla B_{\theta}\right)_{\varphi x}=v$ so that the above is written as $\left(\varphi_{*}\right)_{x}^{*} v=\left(\nabla B_{\Phi^{-1} \theta}\right)_{x}$ and thus from property (v) in Section 4.2 it is concluded that $\left|\left(\varphi_{*}\right)_{x}^{*} v\right|=\left|\left(\nabla B_{\Phi^{-1} \theta}\right)_{x}\right|=1$ which implies that $\left(\varphi_{*}\right)_{x}^{*}$ and consequently $\left(\varphi_{*}\right)_{x}$ is a linear isometry. Since $x \in X$ is arbitrary, $\varphi$ turns out to be an isometry of $(X, g)$. 
To show that $\Phi$ coincides with $\partial X$-extension $\hat{\varphi}$ we make use of (47) together with the following

$$
\left(d B_{\hat{\varphi}^{-1} \theta}\right)_{x}(u) \mu_{\varphi x}=\left(d B_{\theta}\right)_{\varphi x}\left(\left(\varphi_{*}\right)_{x} u\right) \mu_{\varphi x}
$$

which is derived by differentiating the Busemann cocycle formula (28) to get for any $u \in T_{x} X, x \in X$

$$
\left(d B_{\hat{\varphi}^{-1} \theta}\right)_{x}(u)=\left(d B_{\Phi^{-1} \theta}\right)_{x}(u) .
$$

Namely, we have $d\left(B_{\hat{\varphi}^{-1} \theta}-B_{\Phi^{-1} \theta}\right)=0$ on $X$ for any $\theta \in \partial X$. Since $X$ is connected, $B_{\hat{\varphi}^{-1} \theta}(x)-$ $B_{\Phi^{-1} \theta}(x)=C$ for a constant $C$ which depends on $\theta$. From this it follows that $\hat{\varphi}=\Phi$. In fact, assume $\hat{\varphi}^{-1} \theta \neq \Phi^{-1} \theta$ for some $\theta \in \partial X$, otherwise, and let $x \rightarrow \Phi^{-1} \theta$. Then, from the visibility axiom (see Proposition 5) $B_{\hat{\varphi}^{-1} \theta}(x)-B_{\Phi^{-1} \theta}(x) \rightarrow-\infty$ contradicting that $C$ is constant.

\section{Author Contributions}

Mitsuhiro Itoh conceived the idea and analysis. Mitsuhiro Itoh and Hiroyasu Satoh together did derivation and wrote the paper. Both authors have read and approved the final manuscript.

\section{Conflicts of Interest}

The authors declare no conflict of interest.

\section{References}

1. Itoh, M.; Satoh, H. Fisher Information Geometry of The Barycenter Map. AIP Conf. Proc. 2015, 1641, http://dx.doi.org/10.1063/1.4905967.

2. Friedrich, T. Die Fisher-Information und symplektische Strukturen. Math. Nachr. 1991, 153, 273-296.

3. Schoen, R.; Yau, S.-T. Lectures on Differential Geometry; Intern. Press: Boston, MA, USA, 1994.

4. Itoh, M.; Satoh, H. Information geometry of Poisson Kernels on Damek-Ricci spaces. Tokyo J. Math. 2010, 33, 129-144.

5. Itoh, M.; Satoh, H. Fisher Information Geometry, Poisson Kernel and Asymtotical Harmonicity. Differ. Geom. Appl. 2011, 29, S107-S115.

6. Itoh, M.; Shishido, Y. Fisher Information Metric and Poisson Kernels, Differ. Geom. Appl. 2008, 26, 347-356.

7. Manning, A.: Topological entropy for geodesic flows, Ann. Math., 1979, 110, 567-573.

8. Besson, G.; Courtois, G.; Gallot, S. Entropies et Rigidités des espaces localement symétriques de courbure strictement négative. Geom. Func. Anal. 1995, 5, 731-799.

9. Cafaro, C., Ali, S.A.: Jacobi fields on statistical manifolds of negative curvature, Physica, 2007, D234, 70-92.

10. Berndt, J.; Tricerri, F.; Vanhecke, L. Generalized Heisenberg Groups and Damek-Ricci Harmonic Spaces; Lecture Notes in Mathematics, Volume 1598; Springer: Heidelberg, Germany, 1991.

11. Itoh, M.; Satoh, H.; Suh, Y.-J. Horospheres and Hyperbolicity of Hadamard manifolds. Differ. Geom. Appl. 2014, 35, Supplement, 50-68. 
12. Szabo, Z. The Lichnerowicz conjecture on harmonic manifolds. J. Diff. Geom. 1990, 31, 1-28.

13. Heber, J. On harmonic and asymptotically harmonic homogeneous spaces, Geom. Funct. Anal. 2006, 16, 869-890.

14. Amari, S.; Nagaoka, H. Methods of Information Geometry; Translations of Mathematical Monographs, Volume 191; American Mathematical Society: Providence, RI, USA, 2000.

15. Helgason, S. Differential Geometry and Symmetric Spaces; Academic Press: New York, NY, USA, 1962.

16. Douady, E.; Earle, C. Conformally natural extension of homeomorphisms of the circle. Acta Math. 1986, 157, 23-48.

17. Besson, G.; Courtois, G.; Gallot, S. Minimal entropy and Mostow's rigidity theorems. Erg. Th. Dyn. Sys. 1996, 16, 623-649.

18. Arnaudon, M.; Barbaresco, F.; Yang, L. Medians and Means in Riemannian Geometry, Existence, Uniqueness and Computation. In Matrix Information Geometry; Nielson, F., Bhatia, R., Eds.; Springer: Heidelberg, Germany, 2013; pp.169-197.

19. Barbaresco, F.: Information Geometry of Covariance Matrix: Cartan-Siegel Homogeneous Bounded Domains, Mostow/Berger Fibration and Fréchet Median. In Matrix Information Geometry; Nielson, F., Bhatia, R., Eds.; Springer: Heidelberg, Germany, 2013; pp.199-255.

20. Furstenberg, H. A Poisson formula for semi-simple Lie groups. Ann. Math. 1963, 77, 335-386.

21. Eberlein, P.; O’Neill, B. Visibility manifolds. Pac. J. Math. 1973, 46, 45-110.

22. Do Carmo, M. Riemannian Geometry; Birkhäuser: Boston, MA, USA, 1992.

23. Gallot, S.; Hulin, D.; Lafontaine, J. Riemannian Geometry, 2nd ed.; Springer-Verlag: Berlin, Germany, 1980.

24. Itoh, M.; Satoh, H. Information Geometry of Barycenter Map. In Real and Complex Submanifolds; Suh, Y.J., Berndt, J., Ohnita, Y., Kim, B.H., Eds.; Springer Proceedings in Mathematics and Statistics, Volume 106; Springer: Tokyo, Japan, 2014; pp. 79-88.

25. Itoh, M.; Satoh, H. Information Geometry of Busemann-Barycenter for Probability Measures. Int. J. Math. 2015, in press.

26. Halmos, P. Measure Theory; Graduate Texts in Mathematics, Volume 18; Springer: New York, NY, USA, 1950.

27. Villani, C. Topics in Optimal Transformation; Gradudate Study in Mathematics, Volume 58; American Mathematical Society: Providence, RI, USA, 2003.

28. Fathi, A. Structure of the group of homeomorphisms preserving a good measure on a compact manifold. Ann. scient. Éc. Norm. Sup 1980, 13, 45-93.

29. Oxtoby, C.; Ulam, S.M. Measure preserving homeomorphisms and metrical transitivity. Ann. Math. 1941, 42, 874-920.

30. Sakai, T. Riemannian Geometry; American Mathematical Society: Providence, RI, USA, 2000.

31. Ballmann, W.; Gromov, M.; Schroeder, V. Manifolds of Nonpositive Curvature; Progress in Mathematics, Volume 61; Birkhäuser: Boston, MA, USA, 1985.

32. Heintze, E.; Im Hof, H.-C. Geometry of horospheres. J. Diff. Geom. 1977, 12, 481-491.

33. Guivarc'h, Y.; Ji, L.; Taylor, J.C. Compactifications of Symmetric Spaces; Progress in Mathematics, Volume 156; Birkhäuser: Boston, MA, USA, 1997. 
34. Ledrappier, F. Harmonic measures and Bowen-Margulis measures. Israel J. Math. 1990, 71, 275-287.

35. Eberlein, P. Geodesic Flows in Manifolds of Nonpositive Curvature. Proc. Symp. Pure Math. 2001, 69, 525-571.

(c) 2015 by the authors; licensee MDPI, Basel, Switzerland. This article is an open access article distributed under the terms and conditions of the Creative Commons Attribution license (http://creativecommons.org/licenses/by/4.0/). 